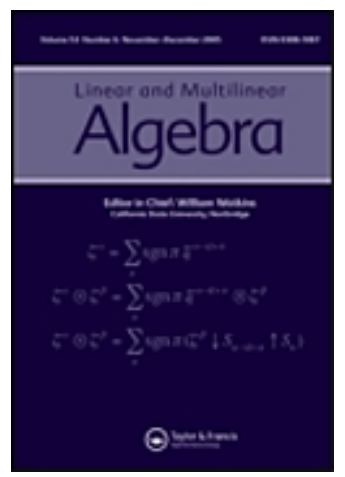

\title{
State Canonical Form for a Class of Uncontrollable Linear Systems
}

\begin{tabular}{|c|c|}
\hline Journal: & Linear and Multilinear Algebra \\
\hline Manuscript ID & 2019-0063.R1 \\
\hline Manuscript Type: & Original Article \\
\hline Date Submitted by the Author: & $n / a$ \\
\hline Complete List of Authors: & $\begin{array}{l}\text { Compta, Albert; Univ. Pol. Catalunya, } \\
\text { Matematica Aplicada I } \\
\text { Ferrer, Josep; Univ. Pol. Catalunya, } \\
\text { Matematica Aplicada I } \\
\text { Peña Carrera, Marta; Universitat Politècnica } \\
\text { de Catalunya }\end{array}$ \\
\hline Keywords: & $\begin{array}{l}\text { Linear control systems, State variables, } \\
\text { Canonical forms, Block lower diagonal } \\
\text { matrices }\end{array}$ \\
\hline $\begin{array}{r}<\mathrm{a} \\
\text { href="http://www.ams.org/mathscinet/msc/msc2010.html" } \\
\text { target="_blank" }>2010 \text { Mathematics Subject } \\
\text { Classification }</ \text { a }>\text { : }\end{array}$ & 15A21, 93B10, 15A03 \\
\hline
\end{tabular}

\section{SCHOLARONE}

Manuscripts 


\title{
State Canonical Form for a Class of Uncontrollable Linear Systems *
}

\author{
Albert Compta, Josep Ferrer and Marta Peña \\ e-mails: albert.compta@upc.edu,josep.ferrer@upc.edu,marta.penya@upc.edu
}

June 3, 2019

\begin{abstract}
We tackle the obtaining of canonical forms for classifying linear control systems with regard to changes in the state variables. Although it was solved in the 80's for controllable systems, it is still an open problem for the general case of multiparametric non-necessarily controllable systems. Here we obtain a general reduced form which is canonical for a class of systems which includes the cases already known (uniparametric systems, controllable systems).
\end{abstract}

\begin{abstract}
AMS Mathematics Subject Classification : 15A21, 93B10, 15A03.
\end{abstract}
Keywords: Linear control systems; State variables; Canonical forms; Block lower diagonal matrices.

\section{Introduction}

The internal representation of linear control systems motivates some different equivalence relations depending on if one considers changes of variables (state, input, output), external injections, feedbacks, and so on. Their study has generated a large literature, mainly in the 70's and 80 's. However, for the apparently simplest equivalence, when only changes in the state space are considered, canonical forms have been obtained only for the cases of uniparametric systems ([1], [4]) or controllable systems ([3]). For the general case, we obtain a reduced form (the MG reduced form) which is canonical for a class of linear systems which includes both already known particular cases above (uniparametric systems, controllable systems): the class when the so-called associated Hermite system is fully decouplable.

We consider pairs of matrices $(A, B) \in M_{N}(\mathbb{C}) \times M_{N \times m}(\mathbb{C})$ representing multiparametric linear control systems and the equivalence relation given by linear changes in the state variables, that is to say, the group action $\left(S^{-1} A S, S^{-1} B\right), S \in M_{N}^{*}(\mathbb{C})$. We can restrict ourselves to the case $A=J$, a nilpotent Jordan matrix, so that we have to study the so-called p-action $L B$, $L \in \mathbb{Z}^{*}(J)=\left\{L \in M_{N}^{*}(\mathbb{C}): L J=J L\right\} \equiv \mathbb{Z}^{*}(p)$, where $p$ is the Segre characteristic of $J$.

\footnotetext{
*Supported by the projects MTM2017-90682-REDT (all authors) and MTM2017-85669-P (third author).
} 
The first step (Definition 4.1) is that one associates to $B=\left(b_{1}, \ldots, b_{m}\right)$ a so-called BLD-matrix $K=\left(K_{1}, \ldots, K_{m}\right)$ where $K_{i}=\left(b_{i}, J b_{i}, \ldots, J^{q_{i}-1} b_{i}\right)$, with $J^{q_{i}-1} b_{i} \neq 0, J^{q_{i}} b_{i}=0, i=1, \ldots, m$ so that the action $L B$ is equivalent to some elementary transformations of $K$, which we call row BLD-ETs (Definition 2.10).

The key point in our approach is introducing the so-called Hermite systems (Definition 5.1) when the columns of $K$ (or equivalently, the non-zero columns of the controllability matrix) are linearly independent. From a control point of view, it means that the controls $b_{1}, \ldots, b_{m}$ are decoupled in the sense that the effect of each control can not be obtained by means of the other ones.

Thus, the second step in our technique is reducing $K$ to a BLD matrix $K_{H}^{\prime}$ (Definition 5.9) corresponding to an Hermite system $\left(J, B_{H}\right)$ by means of a factorization depending on the socalled Hermite coefficients, which are invariant with regard to changes in the state variables. In this way, the p-equivalence class of $K$ is determined (Theorem 5.13) by its Hermite coefficients and the p-class of $K_{H}^{\prime}$ (a nicer class of BLD matrices).

Therefore, row BLD-ETs will be applied to the BLD matrices $K_{H}^{\prime}$ (Theorem 6.1). Indeed, we act successively in each block column of $K_{H}^{\prime}$, following a pattern similar to the MG-algorithm for single block column (Definition 3.2). In this way we obtain (Definition 6.4) the so-called MG reduced form $U+X$ of $K_{H}^{\prime}$, where the non-zero blocks of $U$ are unitary ones placed in different block rows and block columns and, for each unitary block of $U$, possible non-zero blocks of $X$ are placed in the same block row and in the right block columns (see Theorem 6.1). Also we obtain the desired MG reduced form $B_{M G}$ of $B$.

Finally, the reduction process is finished if $X=0$ (which correspond to $K_{H}^{\prime}$ being a fully decouplable system), so that $B_{M G}$ is a canonical form for the considered system (Corollary 7.1). It includes both the particular cases already known: uniparametric systems, controllable systems (Proposition 7.3).

If $X \neq 0$, it gives also a canonical form in some particular cases where no additional reductions are possible. For example, if $X$ has only a non-zero block in each block column and it is a diagonal one (Proposition 8.1). In the general case, further reductions are possible (Theorem 8.7 ), but they do not give a canonical form because some entries are not invariant, even for $m=2$ (Remark 8.8).

The paper is organized as follows. Section 2 contains some preliminaries concerning linear control systems and BLD-matrices. Section 3 is devoted to present the MG-algorithm. In Section 4 we associate a BLD matrix $K$ to each system, in such a way that its p-class is determined by the state equivalence class of the original control system, and conversely. The key point is tackled in Section 5, where Hermite systems are defined, and any other is factorized in an Hermite system and a factor depending on the Hermite coefficients. Next, in Section 6, we obtain the MG reduced form by applying in some sense the MG-algorithm to the Hermite system associated to the given one. In Section 7 we check that the MG reduced form is indeed a canonical form in some cases including the uniparametric systems and the controllable ones (the particular cases nowadays known). Finally, in Section 8 we tackle to generalize our results: great difficulties appear even for the biparametric case.

In all the paper we denote by $M_{N}(\mathbb{C})$ the complex $N$-square matrices and by $M_{N \times d}(\mathbb{C})$ the ones having $N$ rows and $d$ columns. In all the cases, if $M$ is a set of matrices, then $M^{*}$ denotes those having maximal rank and $S p(M)$ denotes the vectorial subspace spanned by the columns of the 
matrices in $M$. Also, we denote by $\underline{d}=\{1,2, \ldots, d\}$ for all integer $d \geq 1$.

\section{Preliminaries}

\subsection{Equivalence between linear control systems}

The behavior of the state variables $x_{1}(t), \ldots, x_{N}(t)$ of a time-invariant linear control system is characterized by the state equation

$$
\dot{x}(t)=A x(t)+B u(t)
$$

where $u(t)=\left(u_{1}(t), \ldots, u_{m}(t)\right)$ are the controls, and $A \in M_{N}(\mathbb{C}), B \in M_{N \times m}(\mathbb{C})$. So, it is characterized by the pair $(A, B)$. Its controllability subspace $V$ is the one spanned by the columns of the so-called controllability matrix $\left(B, A B, \ldots, A^{N-1} B\right)$ and the system is controllable (or reachable) if $\operatorname{dim} V=N$, that is to say, if $\operatorname{rank}\left(B, A B, \ldots, A^{N-1} B\right)=N$. We consider the general case when $\operatorname{rank}\left(B, A B, \ldots, A^{N-1} B\right) \leq N$.

If $A=\operatorname{diag}\left(A_{1}, A_{2}\right)$ and $B=\operatorname{diag}\left(B_{1}, B_{2}\right)$, the subsystems $\left(A_{1}, B_{1}\right)$ and $\left(A_{2}, B_{2}\right)$ do not interact with each other. One says then that $(A, B)$ is decoupled. In particular, $V=V_{1} \oplus V_{2}$, where $V_{1}$ and $V_{2}$ are the controllability subspaces of $\left(A_{1}, B_{1}\right)$ and $\left(A_{2}, B_{2}\right)$, respectively. We are mainly interested in the case when $(A, B)$ is decoupled into as many subsystems as there are controls, that is to say, when each subsystem is uniparametric:

Definition 2.1 Given a system $(A, B)$ as above, if

$$
A=\operatorname{diag}\left(A_{1}, \ldots, A_{m}\right), \quad B=\operatorname{diag}\left(B_{1}, \ldots, B_{m}\right)
$$

where $\left(A_{1}, B_{1}\right), \ldots,\left(A_{m}, B_{m}\right)$ are uniparametric systems, we say that $(A, B)$ is fully decoupled.

A linear change $x=S \bar{x}, S \in M_{N}^{*}(\mathbb{C})$, in the state variables leads to a new state equation

$$
\dot{\bar{x}}(t)=\left(S^{-1} A S\right) \bar{x}(t)+\left(S^{-1} B\right) u(t)
$$

that is to say, to a new pair of matrices $\bar{A}=S^{-1} A S, \bar{B}=S^{-1} B$. The following definition formalizes this transformation:

Definition 2.2 Let us consider pairs of matrices $(A, B), A \in M_{N}(\mathbb{C}), B \in M_{N \times m}(\mathbb{C})$. Two of them $(A, B)$ and $(\bar{A}, \bar{B})$ are called state equivalent (that is to say, with regard to linear changes in the state variables) if and only if there is $S \in M_{N}^{*}(\mathbb{C})$ such that:

$$
\bar{A}=S^{-1} A S, \quad \bar{B}=S^{-1} B .
$$

If $(\bar{A}, \bar{B})$ is fully decoupled for some $S \in M_{N}^{*}(\mathbb{C})$, we will say that $(A, B)$ is fully decouplable.

Remark 2.3 Notice that other equivalence relations can be done, involving outputs, inputs, feedbacks and so on. For example, by considering linear changes in the state variables as in Definition 2.2 and also in the control ones: $\bar{B}=S^{-1} B T$. It is just equivalent to the classification 
of the controllability subspaces $V$ as A-invariant subspaces, which is a "wild" problem (see [2] and [5]). Obviously, this equivalence relation is the same as in Definition 2.2 if $m=1$, that is to say, the classification of monogenic A-invariant subspaces in [1] is equivalent to classify uniparametric systems as in Definition 2.2 (Proposition 4.4).

Clearly, we can reduce the study of this equivalence relation to the case $A=\bar{A}=J$, a nilpotent lower Jordan matrix. Let us write $p=\left(p_{1}, \ldots, p_{n}\right), p_{1} \geq \cdots \geq p_{n}>0$, its Segre characteristic and $\mathbb{Z}^{*}(p)$ the non-singular matrices commuting with $J$. Then, the above equivalence relation can be reduced to the following one:

Definition 2.4 Let $p=\left(p_{1}, \ldots, p_{n}\right)$ be a non-increasing partition of $N$, i.e., $p_{1} \geq \cdots \geq p_{n}>0$ and $p_{1}+\ldots+p_{n}=N$. Two matrices $B, \bar{B} \in M_{N \times m}(\mathbb{C})$ are called $p$-equivalent if

$$
\bar{B}=L B, \quad L \in \mathbb{Z}^{*}(p)
$$

Remark 2.5 Notice that the matrix belonging to $\mathbb{Z}^{*}(p)$ represents a change of Jordan bases of $J$. Hence the action $L B$ is a change of Jordan coordinates of the columns of B. For each Jordan basis of $J$ we write $M_{1}, \ldots, M_{n}$ the monogenic subspaces spanned by the corresponding Jordan chains, so that one has the decomposition $\mathbb{C}^{N}=M_{1} \oplus \cdots \oplus M_{n}$, where the subspaces $M_{i}$ depend on the considered Jordan basis.

Remark 2.6 If convenient, we can consider right multiplications $B R, R \in M_{m}^{*}(\mathbb{C})$, bearing in mind that if $\bar{B}=L(B R)$ is a reduced form of $B R$, then a reduced form of $B$ is just $\bar{B} R^{-1}=L B$. In particular, we can reorder the columns of $B$ by means of $B P$, where $P$ is a permutation matrix. Notice that the final reduced matrix $L B$ will be different for different permutations $P$ (or different right factors $R$ ).

\subsection{The BLD matrices}

By means of associating to each matrix $B=\left(b_{1}, \ldots, b_{m}\right) \in M_{N \times m}(\mathbb{C})$ a so-called BLD matrix $\left(K_{1}, \ldots, K_{m}\right)$, we will characterize the equivalence relation in Definition 2.4 as matrix elementary transformations in the associated BLD matrix. Let us recall some notation and properties of this kind of matrices:

Definition 2.7 (1) A matrix is called lower diagonal (LD) if it is a lower triangular matrix constant along the diagonals.

(2) A partitioned matrix whose blocks are LD matrices will be called block lower diagonal (BLD).

(3) We denote by $B L D(p, q)$ the BLD matrices with respect to the block partition $(p, q)$, that is to say, its rows are partitioned according to $p=\left(p_{1}, \ldots, p_{n}\right)$ and its columns are partitioned according to $q=\left(q_{1}, \ldots, q_{m}\right)$.

(4) If $Y \in B L D(p, q)$, we denote by $Y_{i j}$ the block in $M_{p_{i} \times q_{j}}(\mathbb{C})$, by $D_{i j}^{k}$ the $k$-diagonal of this block, where the first diagonal is the element in the left bottom corner, and by $y_{i j}^{k}$ the entries in $D_{i j}^{k}$. Then $y_{i j}^{k}=0$ if $k>\min \left(p_{i}, q_{j}\right)$. 
(5) In each block $Y_{i j}$, we say that the entry $y_{i j}^{k}$ or the diagonal $D_{i j}^{k}$ has height $k$, depth $\left(p_{i}-k\right)$ and horizontal depth $q_{j}-k$.

(6) If $k_{i j}=\max \left\{k: y_{i j}^{k} \neq 0\right\}$, we say that $k_{i j}$ is the height of $Y_{i j}$ and we refer to the entry $y_{i j}^{k_{i j}}$ or the diagonal $D_{i j}^{k_{i j}}$ as the highest ones.

(7) We define $I_{i j}^{k}=\left(\begin{array}{cc}0 & 0 \\ I_{k} & 0\end{array}\right) \in M_{p_{i} \times q_{j}}(\mathbb{C}) \quad$ for $\quad 1 \leq k \leq \min \left(p_{i}, q_{j}\right)$, so that we have $Y_{i j}=\sum_{1 \leq k \leq k_{i j}} y_{i j}^{k} I_{i j}^{k}$. In particular, if $Y_{i j}=y I_{i j}^{k}$ for some $k$ we say that $Y_{i j}$ is a diagonal block; $a$ unitary block if $y=1$ (an identity block if $k=\min \left(p_{i}, q_{j}\right)$ ). Then, we denote by $e_{i}^{k}$ its first column (which does not depend on $j$ ).

(8) We define a block row and a block column as $Y_{i *}=\left(Y_{i 1}, Y_{i 2}, \ldots, Y_{i m}\right)$ and $Y_{* j}=\left(Y_{1 j}^{t}, Y_{2 j}^{t}, \ldots, Y_{n j}^{t}\right)^{t}$, respectively.

It is well known that:

Lemma 2.8 If $J$ is a nilpotent Jordan matrix with Segre characteristic $p$, then the closed subgroup $\mathbb{Z}^{*}(p)$ of $G l(N)$ formed by the non singular matrices which commute with $J$ is just $B L D^{*}(p, p)$. Thus, the elements $L \in B L D^{*}(p, p)$ are the changes of Jordan bases of $J$ (see Remark 2.5).

For a matrix in $B L D(p, q)$, the left multiplication by $L \in \mathbb{Z}^{*}(p)$ in Definition 2.4 is equivalent to certain elementary transformations, as we precise in the following lemma:

Lemma 2.9 [2] Let $Y \in B L D(p, q)$. The left multiplication by matrices $L \in B L D^{*}(p, p)$ is equivalent to a sequence of the following transformations, for each block row $Y_{i *}$ :

(1) Multiplying $Y_{i *}$ by a non-zero scalar $\beta$.

(2) Adding the first $t$ rows of $\beta Y_{i *}$ (where $\beta$ is an arbitrary scalar) to the last $t$ rows of any $Y_{i^{\prime} *}\left(\right.$ for any $1 \leq i^{\prime} \leq n$ and where $\left.1 \leq t \leq \min \left(p_{i}, p_{i^{\prime}}\right)\right)$.

Definition 2.10 We refer to the transformations above as row BLD elementary transformations (row BLD-ETs).

Remark 2.11 According to the above lemmas, to each row BLD-ET one associates a left multiplication $L B, L \in \mathbb{Z}^{*}(p)$ or, equivalently, a change of the Jordan basis of $J$.

Example 2.12 Let us consider $Y$ below. Then, $L Y$ is obtained by applying (2) with: $t=2$, $\beta=-4, i=1, i^{\prime}=2$.

$$
Y=\left[\begin{array}{lll|ll}
1 & 0 & 0 & 0 & 0 \\
2 & 1 & 0 & 6 & 0 \\
3 & 2 & 1 & 7 & 6 \\
\hline 4 & 0 & 0 & 8 & 0 \\
5 & 4 & 0 & 9 & 8
\end{array}\right] \quad L Y=\left[\begin{array}{ccc|cc}
1 & 0 & 0 & 0 & 0 \\
2 & 1 & 0 & 6 & 0 \\
3 & 2 & 1 & 7 & 6 \\
\hline 0 & 0 & 0 & 8 & 0 \\
-3 & 0 & 0 & -15 & 8
\end{array}\right]
$$


As announced, we will use BLD-ETs in order to simplify the matrices $Y \in B L D(p, q)$. Indeed we will focus mainly on the following techniques.

Proposition 2.13 [2] Let $Y \in B L D(p, q)$

(1) Any block $Y_{i j}$ can be reduced, by means of row BLD-ETs, to the unitary one $I_{i j}^{k_{i j}}$, where $k_{i j}$ is the height of $Y_{i j}$ (see (6) in Definition 2.7).

(2) Let $Y_{i j}=I_{i j}^{k_{i j}}$ be a unitary block. By means of row BLD-ETs one can make 0 all the other diagonals in $Y_{* j}$ having the same or less height than $k_{i j}$, and the same or greater depth than $p_{i}-k_{i j}$ :

$$
\bar{y}_{s j}^{k}=0, \quad \text { if } \quad k \leq k_{i j} \quad \text { and } \quad p_{s}-k \geq p_{i}-k_{i j}
$$

(2') In particular, if $Y_{i j}$ is an identity matrix (that is to say, $k_{i j}=q_{j}=p_{i}$ ), then all the remaining blocks in $Y_{* j}$ can be made 0.

Notice that, in (2) above, the blocks in $Y_{* j^{\prime}}$, for $j^{\prime} \neq j$, remain unaffected if $Y_{i j^{\prime}}=0$, but not in general. Because of that, we must pay attention when BLD-ETs are applied by recurrence with regard to $j$ (for example, in Theorem 6.1).

Definition 2.14 We define by $(i, j)$-row BLD-ET the composition of row BLD-ETs in (1) and (2).

Example 2.15 Let us consider the block $Y_{22}$ in $Y$ below. By means of $(2,2)$-row BLD-ETs as in (1) above, we can make $y_{22}^{1}=0$. Next, by means of $(2,2)$-row BLD-ETs as in (2), we can make $y_{12}^{2}=y_{12}^{1}=y_{32}^{1}=0$, but we cannot change $y_{32}^{2}$.

$$
Y=\left[\begin{array}{ccc|cc|c}
0 & 0 & 0 & 0 & 0 & 0 \\
y_{11}^{3} & 0 & 0 & 0 & 0 & 0 \\
y_{11}^{2} & y_{11}^{3} & 0 & y_{12}^{2} & 0 & 0 \\
y_{11}^{1} & y_{11}^{2} & y_{11}^{3} & y_{12}^{1} & y_{12}^{2} & y_{13}^{1} \\
\hline y_{21}^{3} & 0 & 0 & 0 & 0 & 0 \\
y_{21}^{2} & y_{21}^{3} & 0 & 1 & 0 & 0 \\
y_{21}^{1} & y_{21}^{2} & y_{21}^{3} & y_{22}^{1} & 1 & y_{23}^{1} \\
\hline y_{31}^{2} & 0 & 0 & y_{32}^{2} & 0 & 0 \\
y_{31}^{1} & y_{31}^{2} & 0 & y_{32}^{1} & y_{32}^{2} & y_{33}^{1}
\end{array}\right]
$$

$L Y=\left[\begin{array}{ccc|cc|c}0 & 0 & 0 & 0 & 0 & 0 \\ y_{11}^{3} & 0 & 0 & 0 & 0 & 0 \\ y_{11}^{2} & y_{11}^{3} & 0 & 0 & 0 & 0 \\ y_{11}^{1} & y_{11}^{2} & y_{11}^{3} & 0 & 0 & y_{13}^{1} \\ \hline y_{21}^{3} & 0 & 0 & 0 & 0 & 0 \\ y_{21}^{2} & y_{21}^{3} & 0 & 1 & 0 & 0 \\ y_{21}^{1} & y_{21}^{2} & y_{21}^{3} & 0 & 1 & y_{23}^{1} \\ \hline y_{31}^{2} & 0 & 0 & y_{32}^{2} & 0 & 0 \\ y_{31}^{1} & y_{31}^{2} & 0 & 0 & y_{32}^{2} & y_{33}^{1}\end{array}\right]$

\section{The MG-algorithm}

A basic tool in our technique to reduce $B$ is the following reduction for a block column BLD matrix:

Proposition 3.1 Let us consider a block column BLD matrix $Y=\left(Y_{1}^{t}, \ldots, Y_{n}^{t}\right)^{t} \in B L D(p, q)$, $p=\left(p_{1}, \ldots, p_{n}\right)$. 
(1) There exists a unique matrix $U=\left(U_{1}^{t}, \ldots, U_{n}^{t}\right)^{t}$ of the form $U=L Y$ for some $L \in \mathbb{Z}^{*}(p)$, characterized as follows:

$$
U_{i(s)}=I_{i(s)}^{k(s)}, \quad s \in \underline{r} \quad ; \quad U_{i}=0, \quad i \notin\{i(s): s \in \underline{r}\}
$$

where the finite sequences of indices $i(1), \ldots, i(r)$ and $k(1), \ldots, k(r)$ are such that:

$$
\begin{gathered}
1 \leq i(1)<i(2)<\cdots<i(r) \leq n, \\
q \geq k(1)>k(2)>\cdots>k(r) \geq 1, \\
p_{i(s)} \geq k(s), \quad 1 \leq s \leq r \\
p_{i(s+1)}-k(s+1)<p_{i(s)}-k(s), \quad 1 \leq s \leq r-1
\end{gathered}
$$

(2) In particular, the only non-zero parameters of the first column of $U$ are valued 1 and are placed in the blocks $i(1), \ldots, i(s)$ with $k(1)>\ldots>k(r)$ height and $p_{i(1)}-k(1), \ldots, p_{i(r)}-k(r)$ depth, respectively. As a consequence $p_{i(s)}-p_{i(s+1)} \geq 2,1 \leq s \leq r-1$.

In addition, the matrix $U$ above is the same for any other matrix $\bar{Y}$ obtained from $Y$ by means of row BLD-ETs.

Proof. It is obtained by applying the following algorithm.

(1) Let $k(1)=\max \left\{k_{i}: i \in \underline{n}\right\}$ and $i(1)=\max \left\{i \in \underline{n}: k_{i}=k(1)\right\}$.

Then, $D_{i(1)}^{k(1)}$ is the highest diagonal of $Y_{i(1)}$ and by means of $i(1)$-row BLD-ETs one obtains blocks $U_{1}, \ldots, U_{i(1)}, Y_{i(1)+1}(1), \ldots, Y_{n}(1)$ such that:

(1.1) $U_{i(1)}=I_{i(1)}^{k(1)}$

(1.2) For $i<i(1): U_{i}=0$

(1.3) For $i>i(1)$ : we recall that $D_{i}^{k}=0, k \geq k(1)$, and now $D_{i}^{k}(1)=0$ if $k<k(1)$ and $p_{i}-k \geq p_{i(1)}-k(1)$

(1.3') In particular, $Y_{i}(1)=0$ if $p_{i}=p_{i(1)}$ or $p_{i}=p_{i(1)}-1$

(2) Next, by recurrence, for $s=2, \ldots, r$, let $k(s)=\max \left\{k_{i}: i(s-1)<i \leq n\right\}$ and $i(s)=$ $\max \left\{i: k_{i}=k(s)\right\}$ (obviously $k(s)<k(s-1), i(s)>i(s-1)$ ).

Then, by means of $i(s)$-row BLD-ETs applied to the blocks $Y_{i}$ with $i(s-1)<i \leq n$ one obtains $U_{1}, \ldots, U_{i(1)}, \ldots, U_{i(s)}, Y_{i(s)+1}(s), \ldots, Y_{n}(s)$ such that:

(2.1) $U_{i(s)}=I_{i(s)}^{k(s)}$

(2.2) For $i(s-1)<i<i(s): U_{i}=0$

(2.3) For $i>i(s): D_{i}^{k}(s)=0$ if $k<k(s)$ and $p_{i}-k \geq p_{i(s)}-k(s)$ (or $k \geq k(s)$ )

(2.3') In particular, $Y_{i}(s)=0$ if $p_{i}=p_{i(s)}$ or $p_{i}=p_{i(s)}-1$

It is clear that the indices $i(s), k(s)$ can not be changed by means of row BLD-ETs. So, the matrix $U$ is unique and does not depend on row BLD-ETs in $Y$. 
Definition 3.2 Let us consider a block column BLD matrix $Y \in B L D\left(\left(p_{1}, \ldots, p_{n}\right), q\right)$.

(1) The indices $i(s)$ and $k(s), s \in \underline{r}=\{1, \ldots, r\}$ in the above proposition are called the block indices and the diagonal indices of $Y$, respectively.

(2) The algorithm to obtain them in the proof of this proposition will be called the MGalgorithm.

Example 3.3 Let us consider $p=(7,6,4)$ and the matrix $Y$ below, where $a, b \neq 0$. Clearly $k(1)=5, i(1)=1$.

By means of 1-row BLD-ETs with $D_{1}^{5}$ one obtains the matrix $\bar{Y}$ below.

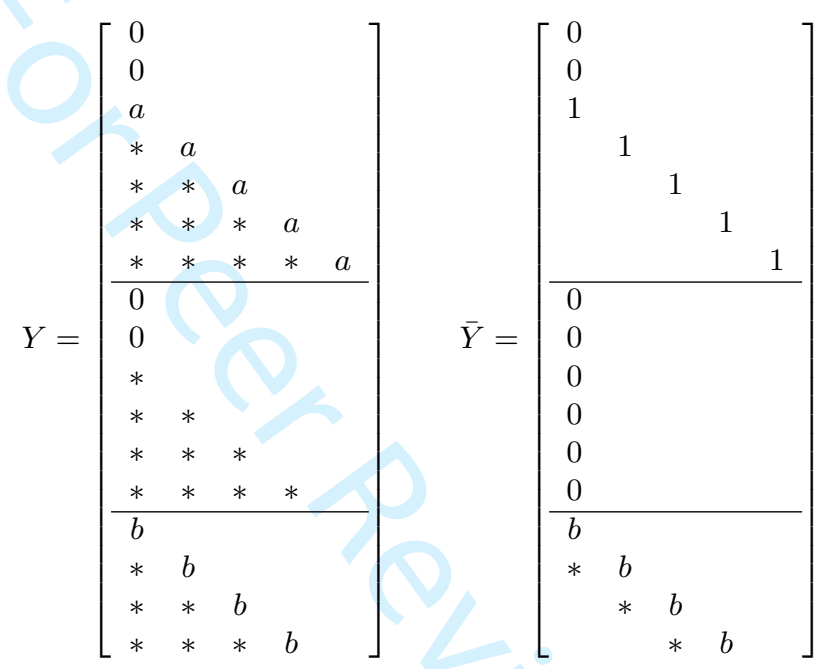

Next $k(2)=4, i(2)=3$ and the bottom block can be reduced to the unitary one $I_{3}^{4}$.

Remark 3.4 In Proposition 4.4 we will see that this algorithm solves immediately the uniparametric case, which is equivalent to the already known classification of monogenic invariant subspaces (Remark 2.3). It justifies its denomination $M G$.

\section{The associated BLD matrix}

We recall that we deal with pairs of matrices $(J, B)$ where $J$ is a nilpotent Jordan matrix with Segre characteristic $p=\left(p_{1}, \ldots, p_{n}\right)$ and $B=\left(b_{1}, \ldots, b_{m}\right)$. Our aim is to obtain a p-equivalent canonical form of it. A key point of our approach is associating to $B$ a BLD matrix and then applying row BLD-ETs based on the MG-algorithm.

Definition 4.1 Let $J \in M_{N}(\mathbb{C})$ be a nilpotent lower Jordan matrix with Segre characteristic $p$ and $B=\left(b_{1}, \ldots, b_{m}\right) \in M_{N \times m}(\mathbb{C})$.

(1) For each $1 \leq j \leq m$, let $K_{j}$ be the matrix whose columns are the non-zero images of $b_{j}$, that is to say, the non-zero columns of the controllability matrix of $\left(J, b_{j}\right)$ :

$$
K_{j}=\left(b_{j}, J b_{j}, \ldots, J^{q_{j}-1} b_{j}\right), \quad J^{q_{j}-1} b_{i} \neq 0, \quad J^{q_{j}} b_{j}=0
$$


They span the controllability subspace $V_{j}$ and: $\quad q_{j}=\operatorname{dim} V_{j}=\operatorname{rank} K_{j}$ We say that $q_{j}$ is the height of $b_{j}$. Obviously, $q_{j} \leq p_{1}$.

(2) We write $q=\left(q_{1}, \ldots, q_{m}\right)$. By reordering the columns of $B$ if necessary (see Remark 2.6), in the sequel we will assume: $\quad p_{1} \geq q_{1} \geq \ldots \geq q_{m}$ where the initial order is preserved if $q_{j}=q_{i}$.

(3) The BLD matrix associated to $B$ is defined as $K=\left(K_{1}, \ldots, K_{m}\right) \in B L D(p, q)$

(4) Conversely, $B=K E$, where $E \in B L D(q,(1, \ldots \underbrace{m}, 1))$ and its only non-zero blocks are the diagonal ones: $\quad E_{j j}=(10 \ldots 0)^{t}$. That is to say, the only non-zero entry of $E_{* 1}, \ldots, E_{* m}$ is valued 1 and placed in the row $1, q_{1}+1, \ldots, q_{1}+\ldots+q_{m-1}+1$, respectively.

(5) The matrices $L K, L \in Z^{*}(p)$, are called p-equivalent to $K$.

The following lemma is obvious:

Lemma 4.2 Let $B, \bar{B} \in M_{N \times m}(\mathbb{C})$ and $K, \bar{K}$ be their associated $B L D$ matrices, respectively. Then:

$\bar{B}=L B$ if and only if $\bar{K}=L K$, where $L \in \mathbb{Z}^{*}(p)$.

Corollary 4.3 Let $B, \bar{B} \in M_{N \times m}(\mathbb{C})$ and $K, \bar{K}$ be their associated $B L D$ matrices, respectively. Then the following two statements are equivalent:

(i) $B, \bar{B}$ are p-equivalent.

(ii) $q=\bar{q}$ and $K, \bar{K}$ are $p$-equivalent.

Therefore, as announced, p-equivalent matrices are obtained by means of row BLD-ETs of $K$ (Lemma 2.9). In particular, the classification for $m=1$ in [1] and [4] follows immediately (see Remark 2.3).

Proposition 4.4 Let us consider the particular case $m=1$, that is to say, $B=\left(b_{1}\right)$ and the associated BLD matrix is a block column $K=\left(K_{1}\right) \in B L D\left(p,\left(q_{1}\right)\right)$. Then:

(i) The $p$-class of $B$ is characterized by the block indices $i(1), \ldots, i(r)$ and the diagonal indices $k(1), \ldots, k(r)$ of $K$.

(ii) A p-canonical form of $B$ is the column matrix described in (2) of Proposition 3.1: the only non-zero parameters are valued 1 and are placed in the blocks $i(1), \ldots, i(s)$ with $k(1)>\ldots>$ $k(r)$ height.

We recall: $\quad p_{i(s+1)}-k(s+1)<p_{i(s)}-k(s)$ and $p_{i(s)}-p_{i(s+1)} \geq 2, \quad 1 \leq s \leq r-1$.

Remark 4.5 The above result gives a geometrical interpretation of the above indices $i(1), \ldots, i(r)$. We recall that the canonical form is the result of a change of the Jordan basis of $J$ (see Remark 2.5). Then, if $M_{1}, \ldots, M_{n}$ are the monogenic Jordan subspaces for the new Jordan basis of $J$, one has

$$
V_{1} \subset M_{i(1)} \oplus \ldots \oplus M_{i(r)}
$$

where $V_{1}$ is the controllability subspace of $\left(J, b_{1}\right)$. 


\section{Reduction to the associated Hermite system having decoupled controls}

A second key tool in our technique is reducing $K$ to a BLD matrix $K_{H}^{\prime}$ whose non-zero columns are linearly independent, by means of a factorization depending on the so-called Hermite coefficients (Definition 5.9). So, the study of the p-equivalence can be reduced to matrices $K_{H}^{\prime}$ of this kind (Theorem 5.13). In the next section the MG-algorithm (Definition 3.2) will be applied to the block columns of such matrices $K_{H}^{\prime}$.

Definition 5.1 Let $J \in M_{N}(\mathbb{C})$ be a nilpotent lower Jordan matrix with Segre characteristic $p$ and $B=\left(b_{1}, \ldots, b_{m}\right) \in M_{N \times m}(\mathbb{C})$. We say that $(J, B)$ is an Hermite system if the columns of the BLD matrix $K$ associated to $B$ (or, equivalently, the non-zero columns of the controllability matrix) are linearly independent. If it is not, the Hermite indices $h_{1}, \ldots, h_{m}$ of $(J, B)$ are defined as:

$$
\begin{gathered}
h_{1}=\min \left\{k: J^{k} b_{1} \in S p\left(b_{1}, \ldots, J^{k-1} b_{1}\right)\right\} \\
h_{j}=\min \left\{k: J^{k} b_{j} \in S p\left(K_{1}, \ldots, K_{j-1}, b_{j}, J b_{j}, \ldots, J^{k-1} b_{j}\right)\right\}, \quad \text { for } \quad 1<j \leq m
\end{gathered}
$$

Obviously, $h_{1}=q_{1}$ and $h_{j} \leq q_{j}$. We write: $h=\left(h_{1}, \ldots, h_{m}\right)$. Notice that it can be non-decreasing (except $h_{1} \geq h_{j}$ ) and that some $h_{j}$ can be 0 .

Proposition 5.2 With the above notation:

(1) $h_{1}=\operatorname{rank} K_{1} ; h_{j}=\operatorname{rank}\left(K_{1}, \ldots, K_{j}\right)-\operatorname{rank}\left(K_{1}, \ldots, K_{j-1}\right), \quad 2 \leq j \leq m$

(2) The chains: $b_{1}, J b_{1}, \ldots, J^{h_{1}-1} b_{1}, b_{2}, J b_{2}, \ldots, J^{h_{2}-1} b_{2}, \ldots, b_{j}, J b_{j}, \ldots, J^{h_{j}-1} b_{j}$ where the corresponding chain is assumed empty if $h_{j}=0$, form a basis of $V_{1}+\ldots+V_{j}$ $\left(=S p\left(K_{1}, \ldots, K_{j}\right)\right)$ for any $1 \leq j \leq m$.

(3) Therefore, for any $1<j \leq m: \quad q_{j}-h_{j}=\operatorname{dim}\left(\left(V_{1}+\ldots+V_{j-1}\right) \cap V_{j}\right)$

Proof.

(1) It follows immediately from the definition.

(2) Clearly, if $J^{h_{j}} b_{j} \in S p\left(K_{1}, \ldots, K_{j-1}, b_{j}, J b_{j}, \ldots, J^{h_{j}-1} b_{j}\right)$

then also $J^{h_{j}+1} b_{j}, \ldots, J^{q_{j}-1} b_{j}$ belong to this subspace. Therefore, the chains $\left(^{*}\right)$ span $V_{1}+$ $\ldots+V_{j}$. And they are linearly independent because, by definition, $h_{j}$ is the minimal exponent verifying $(* *)$.

(3) $\operatorname{dim}\left(\left(V_{1}+\ldots+V_{j-1}\right) \cap V_{j}\right)=\operatorname{dim}\left(V_{1}+\ldots+V_{j-1}\right)+\operatorname{dim} V_{1}-\operatorname{dim}\left(V_{1}+\ldots+V_{j-1}\right)=$ $=\left(h_{1}+\ldots+h_{j}-1\right)+q_{j}-\left(h_{1}+\ldots+h_{j}\right)=q_{j}-h_{j}$.

Remark 5.3 If $h_{j}=0$, then $V_{j} \subset V_{1}+\ldots+V_{j-1}$ and the above formula holds.

If $h_{j}=q_{j}$, then $V_{j} \cap\left(V_{1}+\ldots+V_{j-1}\right)=\{0\} \quad$ so that none of the effects of the control $b_{j}$ can be obtained by means of $b_{1}, \ldots, b_{j-1}$. 
In particular, $(J, B)$ is an Hermite system if $h_{j}=q_{j}$ for all $1 \leq j \leq m$. Then $\quad V_{1} \oplus \ldots \oplus V_{m}$ and we say that the controls $b_{1}, \ldots, b_{m}$ are decoupled in the sense that the effects of each control can not be obtained by means of the other ones.

Thus, we can make the condition in Definition 5.1 explicit.

Definition 5.4 With the notation in Definition 5.1, we define the Hermite coefficients $\lambda_{i j}^{k}$, for $1 \leq j \leq m, 1 \leq i \leq j, 1 \leq k \leq h_{i}$, of $(J, b)$ as the coordinates of $J^{h_{j}} b_{j}$ in the basis (*) from Proposition 5.2:

$$
J^{h_{j}} b_{j}=\sum_{i=1}^{j}\left(\lambda_{i j}^{1} b_{i}+\ldots+\lambda_{i j}^{h_{i}} J^{h_{i}-1} b_{i}\right)
$$

Obviously, all the Hermite coefficients are 0 if $(J, B)$ is an Hermite system. Also, one assumes $\lambda_{i j}^{k}=0$ if $h_{i}=0$.

The following characterization of the Hermite indices (except $\lambda_{i j}^{k}$ for $h_{j}=0$ ) is immediate.

Lemma 5.5 In the basis $\left(^{*}\right)$ from Proposition 5.2, the matrix of the restriction of $J$ to $V_{1}+$ $\ldots+V_{m}$ is an upper triangular $\left(h_{1}, \ldots, h_{m}\right)$-block matrix of the form

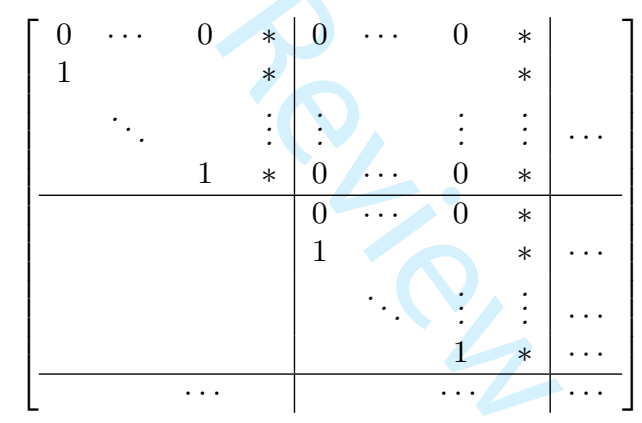

where the entries $*$ in the $h_{1}+\ldots+h_{j}$ column are: $\quad \lambda_{1 j}^{1}, \ldots, \lambda_{1 j}^{h_{1}} ; \lambda_{2 j}^{1}, \ldots, \lambda_{2 j}^{h_{2}} ; \ldots ; \lambda_{j j}^{1}, \ldots, \lambda_{j j}^{h_{j}}$ Again the blocks corresponding to $h_{j}=0$ are empty.

By construction $\lambda_{i j}^{k}=0$ if $i>j, \quad h_{i}=0$ or $k>h_{i}$. Let us see that some other Hermite coefficients are 0 .

Proposition 5.6 With the above notation, let $1 \leq j \leq m$.

(1) For $i=j$, the Hermite coefficients are $0: \quad \lambda_{j j}^{1}=\ldots=\lambda_{j j}^{h_{j}}=0$

(2) More generally, for $1 \leq i \leq j: \quad \lambda_{i j}^{k}=0 \quad$ if $\quad k \leq q_{i}-q_{j}+h_{j}$ In other words, only the following $\left(q_{j}-h_{j}\right)-\left(q_{i}-h_{i}\right)$ Hermite coefficients can be non-zero:

$$
\lambda^{q_{i}-q_{j}+h_{j}+1}, \ldots, \lambda^{h_{i}}
$$

(2') In particular, $\quad \lambda_{i j}^{k}=0 \quad$ if $\quad q_{j}-h_{j} \leq q_{i}-h_{i}$ 
Proof.

(1) Obviously $\lambda_{j j}^{k}$ if $h_{j}=0$. If not, they appear in the diagonal blocks in Lemma 5.5: they must be 0 because the restriction of $J$ to $V_{1}+\ldots+V_{m}$ is nilpotent.

(2) As $J^{q_{j}-h_{j}}\left(J^{h_{j}} b_{j}\right)=J^{q_{j}} b_{j}=0$, one has: $\quad 0=\sum_{i=1}^{j} \sum_{k=1}^{h_{i}} \lambda_{i j}^{k} J^{k-1+q_{j}-h_{j}} b_{j}$

From the linear independence of the vectors in $(*)$ from Proposition 5.2:

$$
\lambda_{i j}^{k}=0 \quad \text { if } \quad k-1+q_{j}-h_{j}<q_{i}
$$

(2') $\lambda_{i j}^{k}=0$ except for $k$ such that: $\quad q_{i}-q_{j}+h_{j}<k \leq h_{i}$.

Example 5.7 For $q=(5,4,2)$ and $h=(5,2,1)$ the only (possible) non-zero Hermite coefficients are: $\lambda_{12}^{4}, \lambda_{12}^{5} ; \lambda_{13}^{5}$.

As a direct consequence of (1) in Proposition 5.6, we have a new characterization of the Hermite indices:

Corollary 5.8 In the conditions of Definition 5.1:

$$
h_{j}=\min \left\{k: J^{k} b_{j} \in S p\left(K_{1}, \ldots, K_{j-1}\right)=V_{1}+\ldots+V_{j-1}\right\}
$$

for $1<j \leq m\left(\right.$ We recall $\left.h_{1}=q_{1}\right)$.

It will be convenient to organize the Hermite coefficients in a BLD matrix $\Lambda$ as follows, in order to obtain the associate Hermite system:

Definition 5.9 Let $J \in M_{N}(\mathbb{C})$ be a nilpotent lower Jordan matrix with Segre characteristic $p=\left(p_{1}, \ldots, p_{n}\right), B=\left(b_{1}, \ldots, b_{m}\right) \in M_{N \times m}(\mathbb{C})$ and $\lambda_{i j}^{k}$ the Hermite coefficients of $(J, B)$, where $1 \leq j \leq m, 1 \leq i<j, q_{i}-q_{j}+h_{j}<k \leq h_{i}$.

(1) We define the matrix of Hermite coefficients $\Lambda \in B L D(q, q)$ of $(J, B)$ as follows:

- the diagonal blocks, as well as the lower ones, are $0: \Lambda_{i j}=0$ if $i \geq j$

- also, for $i<j: \quad \Lambda_{i j}=0 \quad$ if $\quad q_{j}-h_{j} \leq q_{i}-h_{i}$ (in particular, if $h_{i}=0$ )

- if $i<j$ and $q_{j}-h_{j}>q_{i}-h_{i}$, the last row of $\Lambda_{i j}$ is formed by 0 entries and the (possible) non-zero Hermite coefficients (see (2) in Proposition 5.6):

$$
\left(\begin{array}{llllll}
0 & \cdots & 0 & \lambda_{i j}^{h_{i}} & \cdots & \lambda_{i j}^{q_{i}-q_{j}+h_{j}+1}
\end{array}\right)
$$

(2) Then, we associate the system $\left(J, B_{H}\right)$ to $(J, B)$ :

$$
\begin{gathered}
K_{H}^{\prime}=\left(K_{1}^{\prime}, \ldots, K_{m}^{\prime}\right) \doteq K(I-\Lambda) \\
B_{H}=\left(b_{1}^{\prime}, \ldots, b_{m}^{\prime}\right) \doteq K_{H}^{\prime} E=K(I-\Lambda) E
\end{gathered}
$$

which we call the Hermite system associated to $(J, B)$. 
Example 5.10 In the conditions of Example 5.7:

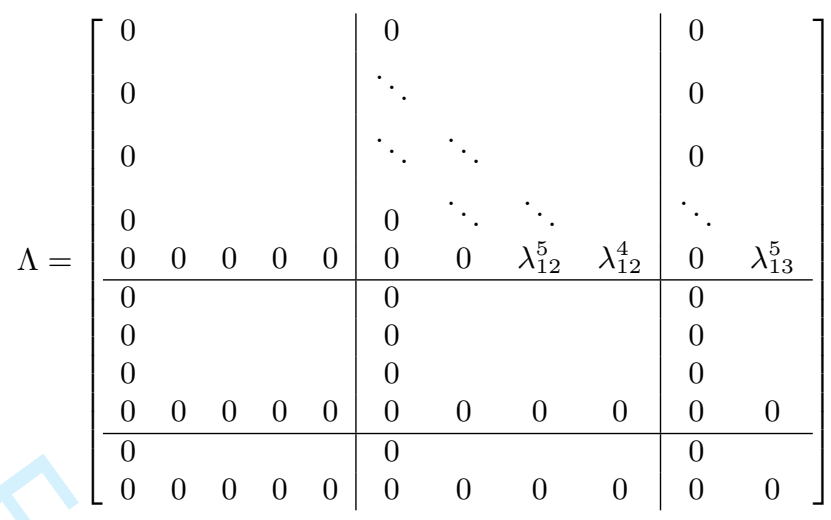

Obviously, if $(J, B)$ is an Hermite system then $\Lambda=0, K_{H}^{\prime}=K$ and $B_{H}=B$. If it is not, let us check that the system $\left(J, B_{H}\right)$ above is indeed an Hermite system.

Lemma 5.11 With the above notation:

(1) For each $K_{j}^{\prime}, 1 \leq j \leq m$, with $h_{j}<q_{j}$, the columns $h_{j}+1, \ldots, q_{j}$ are 0 (in particular, $K_{j}^{\prime}=0$ if $\left.h_{j}=0\right)$.

(2) The remaining columns of $K_{H}^{\prime}$ (that is to say, the first $h_{j}$ columns of each $K_{j}^{\prime}$ ) form the BLD matrix $K_{H}$ associated to $\left(J, B_{H}\right)$ and they are linearly independent. Hence, $K_{H} \in B L D^{*}(p, h)$.

Proof. By construction: $\quad b_{j}^{\prime}=b_{j}-\sum_{i=1}^{j} \sum_{k=q_{i}-q_{j}+h_{j}+1}^{h_{i}} \lambda_{i j}^{k} J^{k-1-h_{j}} b_{i}$.

Hence $\quad J^{h_{j}} b_{j}^{\prime}=J^{h_{j}} b_{j}-\sum_{i} \sum_{k} \lambda_{i j}^{k} J^{k-1} b_{i}=0$.

And analogously for (2).

Remark 5.12 Notice that $K_{H}$ is just a basis of the controllability subspace $V$, presented as in [6].

We attempt the key result in this section: two systems are p-equivalent if they have the same matrices of Hermite coefficients and their associated Hermite systems are p-equivalent.

Theorem 5.13 Let $J \in M_{N}(\mathbb{C})$ be a nilpotent lower Jordan matrix, with Segre characteristic $p$, and $B, \bar{B} \in M_{N \times m}(\mathbb{C})$. Let $\Lambda, \bar{\Lambda}$ be respectively the matrices of Hermite coefficients in Definition 5.9 , and $K_{H}^{\prime}, \bar{K}_{H}^{\prime}$ be respectively the associated Hermite systems. Then the following statements are equivalent:

(i) $B, \bar{B}$ are p-equivalent.

(ii) $\Lambda=\bar{\Lambda}$ and $K_{H}^{\prime}, \bar{K}_{H}^{\prime}$ are p-equivalent. 
Proof.

If $\bar{B}=L B$, then $\Lambda=\bar{\Lambda}$ because the Hermite indices and the Hermite coefficients do not depend on the Jordan basis. Therefore by Corollary $4.3, \bar{K}=L K$ and $\bar{K}_{H}^{\prime}=\bar{K}(I-\Lambda) E=L K_{H}^{\prime}$.

Conversely, $\bar{B}=\bar{K}_{H}^{\prime}(I-\Lambda)^{-1} E=L K_{H}^{\prime}(I-\Lambda)^{-1} E=L B$.

\section{The MG reduced form}

We have seen in the previous section that, in order to classify a system $(J, B)$, it is sufficient to apply row BLD-ETs to the associated matrix $K_{H}^{\prime}$ which verifies the nice properties in Lemma 5.11. Thus,by applying the MG-algorithm in Section 3 to each block column of $K_{H}^{\prime}$, we reduced it to its so-called MG reduced form $U+X$ such that: the non-zero blocks of $U$ are unitary ones, placed in different block rows and block columns; the non-zero blocks of $X$ are placed in block rows having unitary blocks of $U$ in some previous block column. More explicitly:

Theorem 6.1 Given $J \in M_{N}(\mathbb{C})$ a nilpotent lower Jordan matrix with Segre characteristic $p=\left(p_{1}, \ldots, p_{n}\right)$ and $B \in M_{N \times m}(\mathbb{C})$. Let $K_{H}^{\prime}$ be the associated BLD matrix in Definition 5.9.

Then, there is an unique matrix

$$
U+X=L(m, \ldots, 1) K_{H}^{\prime}, \quad L(m, \ldots, 1) \in \mathbb{Z}^{*}(p)
$$

characterized as follows:

(1) For each $1 \leq j \leq m$ :

$$
\begin{gathered}
U_{i j}=0 \quad \text { if } \quad i \neq i_{j}(1), \ldots, i_{j}\left(r_{j}\right) \\
U_{i_{j}(s), j}=I_{i_{j}(s), j}^{k_{j}(s)}, \quad 1 \leq s \leq r_{j}
\end{gathered}
$$

where the finite sequences of block indices $i_{j}(1), \ldots, i_{j}\left(r_{j}\right)$ and diagonal indices $k_{j}(1), \ldots, k_{j}\left(r_{j}\right)$ satisfy:

$$
\begin{gathered}
i_{j}(s) \notin\left\{i_{1}(1), \ldots, i_{1}\left(r_{1}\right)\right\} \cup \ldots \cup\left\{i_{j-1}(1), \ldots, i_{j-1}\left(r_{j-1}\right)\right\}, \quad 1 \leq s \leq r_{j} \\
1 \leq i_{j}(1)<\ldots<i_{j}\left(r_{j}\right) \leq n \\
h_{j} \geq k_{j}(1)>\ldots>k_{j}\left(r_{j}\right) \geq 1 \\
p_{i_{j}(s)} \geq k_{j}(s), \quad 1 \leq s \leq r_{j} \\
p_{i_{j}(s+1)}-k_{j}(s+1)<p_{i_{j}(s)}-k_{j}(s), \quad \text { for } \quad 1 \leq s<r_{j}
\end{gathered}
$$

As a consequence: $\quad p_{i_{j}(s)}-p_{i_{j}(s+1)} \geq 2, \quad 1 \leq s<r_{j}$

(2) For each $1 \leq j \leq m$ :

$$
X_{i j}=0 \quad \text { if } \quad i \neq i_{u}(s) \quad \text { for } \quad \text { all } \quad 1 \leq u<j, \quad 1 \leq s \leq r_{u}
$$

If $\quad X_{i_{u}(s), j} \neq 0$, its height $l_{i_{u}(s), j}$ satisfies $l_{i_{u}(s), j} \leq h_{j}$ and

$$
p_{i_{u}(s)}-l_{i_{u}(s), j}<p_{i_{j}(t)}-k_{j}(t) \quad \text { or } \quad l_{i_{u}(s), j}>k_{j}(t) \quad \text { for } \quad \text { all } \quad 1 \leq t \leq r_{j}
$$

In particular, $X_{* 1}=0$. 
(3) $h_{1}=k_{1}(1) \quad$ and $\quad h_{j}=\max \left(k_{j}(1), \max \left\{l_{i_{u}(s), j}: 1 \leq u<j, 1 \leq s \leq r_{u}\right\}\right)$ for $2 \leq j \leq m$.

Proof.

(1) It is sufficient to apply the MG-algorithm successively: firstly, to the first block column $K_{* 1}$; next, to the blocks of $K_{* 2}$ which correspond to zero blocks in the above transformation; and so on.

(2) By means of $\left(i_{j}(t), j\right)$-row BLD-ET, $1 \leq t \leq r_{j}$ we reduce all $X_{i_{u}(s), j}$ to 0 except those in (2).

(3) The equalities are true because $K_{H}$ has maximal rank, so that it is so the successive transformations of $K_{H}$.

Example 6.2 Let us consider $m=3, n=6$, and the following sequences of block indices:

$$
i_{1}(1)=2, i_{1}(2)=6 \quad\left(r_{1}=2\right) ; \quad i_{2}(1)=1, i_{2}(2)=4 \quad\left(r_{2}=2\right) ; \quad i_{3}(1)=5 \quad\left(r_{3}=1\right)
$$

Then, the matrix $U+X$ in the above theorem has the form:

$$
U+X=\left[\begin{array}{c|c|c}
0 & I_{1,2}^{k_{2}(1)} & X_{1,2} \\
\hline I_{2,1}^{k_{1}(1)} & X_{2,2} & X_{2,3} \\
\hline 0 & 0 & 0 \\
\hline 0 & I_{4,2}^{k_{2}(2)} & X_{4,3} \\
\hline 0 & 0 & I_{5,3}^{k_{1}(3)} \\
\hline I_{6,1}^{k_{1}(2)} & X_{6,2} & X_{6,3}
\end{array}\right]
$$

Summarizing, one has:

Corollary 6.3 Let $J \in M_{N}(\mathbb{C})$ be a nilpotent lower Jordan matrix, with Segre characteristic $p=\left(p_{1}, \ldots, p_{n}\right)$, and $B=\left(b_{1}, \ldots, b_{m}\right) \in M_{N \times m}(\mathbb{C})$. In order to characterize the p-equivalence class of $B$, the following elements are invariant:

(i) the heights $q_{1} \geq \ldots \geq q_{m}$

(ii) the matrix $\Lambda$ (or, equivalently, the Hermite indices $h_{1}, \ldots, h_{m}$ and the Hermite coefficients $\lambda_{i j}^{k}$ )

(iii) the matrix $U$ in the above theorem (or, equivalently, the block indices $i_{j}(s)$ and the diagonal indices $k_{j}(s)$ )

Then, a reduced form for $B$ is $\quad B_{M G} \doteq(U+X)(I-\Lambda)^{-1} E$ where $U+X=L(m, \ldots, 1) K_{H}^{\prime}$ in the above theorem.

Definition 6.4 In the conditions of the above theorem, we say that $U+X=L(m, \ldots, 1) K_{H}^{\prime}$ is the $\mathrm{MG}$ reduced form of $K_{H}^{\prime}$ and that $U$ is its m-monogenic component.

Moreover, we say that the matrix

$$
B_{M G}=(U+X)(I-\Lambda)^{-1} E
$$

is the MG reduced form of $B$. Notice that $(I-\Lambda)^{-1}=I+\Lambda+\ldots \Lambda^{m-1}$ because $\Lambda^{m}=0$. 
In the next section we analize the case $X=0$ when the reduction process is finished. Further reductions are needed if $X \neq 0$ (see Section 8).

Remark 6.5 From a geometrical point of view, the block indices in Theorem 6.1 give a direct decomposition (see Remark 2.5) of the space:

$$
\mathbb{C}^{N}=M^{0} \oplus M^{1} \oplus \ldots \oplus M^{m}
$$

where:

$$
\begin{gathered}
M^{j}=M_{i_{j}(1)} \oplus \ldots \oplus M_{i_{j}\left(r_{j}\right)}, \quad 1 \leq j \leq m \\
M^{0}=\oplus_{i \neq i_{j}(s)} M_{i}
\end{gathered}
$$

From the above theorem, we have the following decomposition for each $b_{j}^{\prime}, 1 \leq j \leq m$ :

$$
b_{j}^{\prime}=u_{j}+x_{j}, \quad u_{j} \in M^{j}, \quad x_{j} \in M^{1} \oplus \ldots \oplus M^{j-1}
$$

The first column of $U_{* j}$ is a canonical form for the component $u_{j}$, as in (ii) of Proposition 4.4 or, equivalently, as generator of a monogenic A-invariant subspace (see Remark 2.3). Also we say that $u_{j}$ is the m-monogenic component of $b_{j}^{\prime}, 1 \leq j \leq m$, and that the block indices $k_{j}(s)$ are the m-monogenic indices of $B$.

On the contrary, the first column of $X_{* j}$ is not a canonical form of the component $x_{j}$ of $b_{j}^{\prime}$, so that further row BLD-ETs are needed if $x_{j} \neq 0$.

\section{The case when the associated Hermite system is fully decou- plable $(X=0)$}

Let us summarize the process till now. Given a system $(J, B)$, in Section 4 we have associated to it a BLD matrix $K$ in such a way that the p-reductions of $B$ can be obtained by means of row BLD-ETs in $K$ (in particular, the MG-algorithm in Section 3). Next, in Section 5, we restrict our study to the associated matrix $K_{H}^{\prime}$ verifying the nice property that it corresponds to an Hermite system. Then, in Section 6 , the matrix $K_{H}^{\prime}$ has been reduced by means of row BLD-ETs (based in the MG-algorithm in Section 3) to a matrix $U+X$. If $X=0$, the reduction process is finished, so that $B_{M G}$ is a canonical form. We will see that this case includes the results already known.

Corollary 7.1 Let us consider $(J, B)$ a system where $J \in M_{N}(\mathbb{C})$ is a nilpotent lower Jordan matrix, with Segre characteristic $p=\left(p_{1}, \ldots, p_{n}\right)$, and $B \in M_{N \times m}(\mathbb{C})$. Let $K$ be its associated $B L D$ matrix, $\Lambda$ be its matrix of Hermite coefficients, $K_{H}^{\prime}=K(I-\Lambda)$ and $U+X=L(m, \ldots, 1) K_{H}^{\prime}$ be its $M G$ reduced form. Then:

(1) $X=0$ if and only if the Hermite system $\left(J, B_{H}\right)$ associated to $(J, B)$ is fully decouplable.

(2) Then, the p-equivalence class of $B$ is characterized by:

(i) the heights $q_{1} \geq \ldots \geq q_{m}$

(ii) the matrix $\Lambda$ of Hermite coefficients (or, equivalently, the Hermite indices $h_{1}, \ldots, h_{m}$ and the Hermite coefficients $\lambda_{i j}^{k}, 1 \leq j \leq m, 1 \leq i<j, q_{i}-q_{j}+h_{j}<k \leq h_{i}$ ) 
(iii) the m-monogenic component $U$ (or, equivalently, m-monogenic indices $i_{j}(1), \ldots i_{j}\left(r_{j}\right)$, $\left.k_{j}(1), \ldots, k_{j}\left(r_{j}\right), 1 \leq j \leq m\right)$

and a p-canonical form of $B$ is

$$
B_{M G}=U(I-\Lambda)^{-1} E
$$

Proof.

If $X=0$, it is clear that the block column $U_{* j}, 1 \leq j \leq m$, is the BLD matrix associated to the subsystem $\left(J^{j}, \overline{b_{j}^{\prime}}\right)$ where $J^{j}=\operatorname{diag}\left(J_{i_{j}(1)}, \ldots, \bar{J}_{i_{j}\left(r_{j}\right)}\right)$ and that all these subsystems are decoupled.

Conversely, if $\left(J, B_{H}\right)$ is fully decouplable, the MG reduction in Theorem 6.1 acts separately in each uniparametric subsystem, giving only unitary blocks.

Then, (2) follows immediately.

Example 7.2 Let us consider $p=(8,4,3)$ and $B$ below. Then $q=(7,5)$ and $K$ is as follows.

$$
B=\left[\begin{array}{c|c}
0 & 0 \\
1 & 0 \\
2 & 0 \\
-1 & -3 \\
0 & -4 \\
5 & 9 \\
1 & -2 \\
-1 & -17 \\
\hline 0 & 0 \\
3 & 1 \\
0 & 2 \\
-1 & -9 \\
\hline 4 & 0 \\
5 & 0 \\
-1 & -12
\end{array}\right], \quad K=\left[\begin{array}{ccccccc|ccccc}
0 & 0 & 0 & 0 & 0 & 0 & 0 & 0 & 0 & 0 & 0 & 0 \\
1 & 0 & 0 & 0 & 0 & 0 & 0 & 0 & 0 & 0 & 0 & 0 \\
2 & 1 & 0 & 0 & 0 & 0 & 0 & 0 & 0 & 0 & 0 & 0 \\
-1 & 2 & 1 & 0 & 0 & 0 & 0 & -3 & 0 & 0 & 0 & 0 \\
0 & -1 & 2 & 1 & 0 & 0 & 0 & -4 & -3 & 0 & 0 & 0 \\
5 & 0 & -1 & 2 & 1 & 0 & 0 & 9 & -4 & -3 & 0 & 0 \\
1 & 5 & 0 & -1 & 2 & 1 & 0 & -2 & 9 & -4 & -3 & 0 \\
-1 & 1 & 5 & 0 & -1 & 2 & 1 & -17 & -2 & 9 & -4 & -3 \\
\hline 0 & 0 & 0 & 0 & 0 & 0 & 0 & 0 & 0 & 0 & 0 & 0 \\
3 & 0 & 0 & 0 & 0 & 0 & 0 & 1 & 0 & 0 & 0 & 0 \\
0 & 3 & 0 & 0 & 0 & 0 & 0 & 2 & 1 & 0 & 0 & 0 \\
-1 & 0 & 3 & 0 & 0 & 0 & 0 & -9 & 2 & 1 & 0 & 0 \\
\hline 4 & 0 & 0 & 0 & 0 & 0 & 0 & 0 & 0 & 0 & 0 & 0 \\
5 & 4 & 0 & 0 & 0 & 0 & 0 & 0 & 0 & 0 & 0 & 0 \\
-1 & 5 & 4 & 0 & 0 & 0 & 0 & -12 & 0 & 0 & 0 & 0
\end{array}\right] .
$$

The two last columns of $K$ are linear dependent from the preceding ones, so that $(J, B)$ is not an Hermite system. We obtain $\left(J, B_{H}\right)$ by means of:

$$
\Lambda=\left[\begin{array}{ccccccc|ccccc}
0 & 0 & 0 & 0 & 0 & 0 & 0 & 0 & 0 & 0 & 0 & 0 \\
0 & 0 & 0 & 0 & 0 & 0 & 0 & 0 & 0 & 0 & 0 & 0 \\
0 & 0 & 0 & 0 & 0 & 0 & 0 & -3 & 0 & 0 & 0 & 0 \\
0 & 0 & 0 & 0 & 0 & 0 & 0 & 2 & -3 & 0 & 0 & 0 \\
0 & 0 & 0 & 0 & 0 & 0 & 0 & 0 & 2 & -3 & 0 & 0 \\
0 & 0 & 0 & 0 & 0 & 0 & 0 & 0 & 0 & 2 & -3 & 0 \\
0 & 0 & 0 & 0 & 0 & 0 & 0 & 0 & 0 & 0 & 2 & -3 \\
\hline 0 & 0 & 0 & 0 & 0 & 0 & 0 & 0 & 0 & 0 & 0 & 0 \\
0 & 0 & 0 & 0 & 0 & 0 & 0 & 0 & 0 & 0 & 0 & 0 \\
0 & 0 & 0 & 0 & 0 & 0 & 0 & 0 & 0 & 0 & 0 & 0 \\
0 & 0 & 0 & 0 & 0 & 0 & 0 & 0 & 0 & 0 & 0 & 0 \\
0 & 0 & 0 & 0 & 0 & 0 & 0 & 0 & 0 & 0 & 0 & 0
\end{array}\right], K_{H}^{\prime}=\left[\begin{array}{cccccccc|ccccc}
0 & 0 & 0 & 0 & 0 & 0 & 0 & 0 & 0 & 0 & 0 & 0 \\
1 & 0 & 0 & 0 & 0 & 0 & 0 & 0 & 0 & 0 & 0 & 0 \\
2 & 1 & 0 & 0 & 0 & 0 & 0 & 0 & 0 & 0 & 0 & 0 \\
-1 & 2 & 1 & 0 & 0 & 0 & 0 & 0 & 0 & 0 & 0 & 0 \\
0 & -1 & 2 & 1 & 0 & 0 & 0 & 0 & 0 & 0 & 0 & 0 \\
5 & 0 & -1 & 2 & 1 & 0 & 0 & 2 & 0 & 0 & 0 & 0 \\
1 & 5 & 0 & -1 & 2 & 1 & 0 & 0 & 2 & 0 & 0 & 0 \\
-1 & 1 & 5 & 0 & -1 & 2 & 1 & -2 & 0 & 2 & 0 & 0 \\
\hline 0 & 0 & 0 & 0 & 0 & 0 & 0 & 0 & 0 & 0 & 0 & 0 \\
3 & 0 & 0 & 0 & 0 & 0 & 0 & 1 & 0 & 0 & 0 & 0 \\
0 & 3 & 0 & 0 & 0 & 0 & 0 & 2 & 1 & 0 & 0 & 0 \\
-1 & 0 & 3 & 0 & 0 & 0 & 0 & 0 & 2 & 1 & 0 & 0 \\
\hdashline 4 & 0 & 0 & 0 & 0 & 0 & 0 & 0 & 0 & 0 & 0 & 0 \\
5 & 4 & 0 & 0 & 0 & 0 & 0 & 0 & 0 & 0 & 0 & 0 \\
-1 & 5 & 4 & 0 & 0 & 0 & 0 & 0 & 0 & 0 & 0 & 0
\end{array}\right]
$$

Finally, by applying the MG-algorithm to $K_{H}^{\prime}$ we obtain $U+X$ below. As $X=0$ we can compute the canonical form $B_{M G}$ : 


$$
U+X=\left[\begin{array}{ccccccc|ccccc}
0 & 0 & 0 & 0 & 0 & 0 & 0 & 0 & 0 & 0 & 0 & 0 \\
1 & 0 & 0 & 0 & 0 & 0 & 0 & 0 & 0 & 0 & 0 & 0 \\
0 & 1 & 0 & 0 & 0 & 0 & 0 & 0 & 0 & 0 & 0 & 0 \\
0 & 0 & 1 & 0 & 0 & 0 & 0 & 0 & 0 & 0 & 0 & 0 \\
0 & 0 & 0 & 1 & 0 & 0 & 0 & 0 & 0 & 0 & 0 & 0 \\
0 & 0 & 0 & 0 & 1 & 0 & 0 & 0 & 0 & 0 & 0 & 0 \\
0 & 0 & 0 & 0 & 0 & 1 & 0 & 0 & 0 & 0 & 0 & 0 \\
0 & 0 & 0 & 0 & 0 & 0 & 1 & 0 & 0 & 0 & 0 & 0 \\
\hline 0 & 0 & 0 & 0 & 0 & 0 & 0 & 0 & 0 & 0 & 0 & 0 \\
0 & 0 & 0 & 0 & 0 & 0 & 0 & 1 & 0 & 0 & 0 & 0 \\
0 & 0 & 0 & 0 & 0 & 0 & 0 & 0 & 1 & 0 & 0 & 0 \\
0 & 0 & 0 & 0 & 0 & 0 & 0 & 0 & 0 & 1 & 0 & 0 \\
\hline 1 & 0 & 0 & 0 & 0 & 0 & 0 & 0 & 0 & 0 & 0 & 0 \\
0 & 1 & 0 & 0 & 0 & 0 & 0 & 0 & 0 & 0 & 0 & 0 \\
0 & 0 & 1 & 0 & 0 & 0 & 0 & 0 & 0 & 0 & 0 & 0
\end{array}\right], \quad B_{M G}=\left[\begin{array}{c|c}
0 & 0 \\
1 & 0 \\
0 & 0 \\
0 & -3 \\
0 & 2 \\
0 & 0 \\
0 & 0 \\
0 & 0 \\
\hline 0 & 0 \\
0 & 1 \\
0 & 0 \\
0 & 0 \\
\hline 1 & 0 \\
0 & 0 \\
0 & -3
\end{array}\right]
$$

More explicitly, the action of the MG-algorithm transforms $B_{H}^{\prime}=\left(b_{1}^{\prime}, b_{2}^{\prime}\right)$ successively as follows:

$\left[\begin{array}{cc}0 & 0 \\ 1 & 0 \\ 2 & 0 \\ -1 & 0 \\ 0 & 0 \\ 5 & 2 \\ 1 & 0 \\ -1 & -2 \\ 0 & 0 \\ 3 & 1 \\ 0 & 2 \\ -1 & 0 \\ 4 & 0 \\ 5 & 0 \\ -1 & 0\end{array}\right],\left[\begin{array}{cc}0 & 0 \\ 1 & 0 \\ 0 & 0 \\ -5 & 0 \\ 2 & 0 \\ 5 & 2 \\ -9 & -4 \\ -3 & -2 \\ 0 & 0 \\ 3 & 1 \\ 0 & 2 \\ -1 & 0 \\ 4 & 0 \\ 5 & 0 \\ -1 & 0\end{array}\right],\left[\begin{array}{cc}0 & 0 \\ 1 & 0 \\ 0 & 0 \\ 0 & 0 \\ 2 & 0 \\ -20 & 2 \\ 1 & -4 \\ 22 & 8 \\ 0 & 0 \\ 3 & 1 \\ 0 & 2 \\ -1 & 0 \\ 4 & 0 \\ 5 & 0 \\ -1 & 0\end{array}\right],\left[\begin{array}{cc}0 & 0 \\ 1 & 0 \\ 0 & 0 \\ 0 & 0 \\ 0 & 0 \\ -20 & 2 \\ 1 & -4 \\ 18 & 8 \\ 0 & 0 \\ 3 & 1 \\ 0 & 2 \\ -1 & 0 \\ 4 & 0 \\ 5 & 0 \\ -1 & 0\end{array}\right],\left[\begin{array}{ll}0 & 0 \\ 1 & 0 \\ 0 & 0 \\ 0 & 0 \\ 0 & 0 \\ 0 & 2 \\ 0 & -4 \\ 0 & 8 \\ 0 & 0 \\ 0 & 1 \\ 0 & 2 \\ 0 & 0 \\ 1 & 0 \\ 0 & 0 \\ 0 & 0\end{array}\right],\left[\begin{array}{cc}0 & 0 \\ 1 & 0 \\ 0 & 0 \\ 0 & 0 \\ 0 & 0 \\ 0 & 2 \\ 0 & -4 \\ 0 & 8 \\ 0 & 0 \\ 0 & 1 \\ 0 & 0 \\ 0 & 0 \\ 1 & 0 \\ 0 & 0 \\ 0 & 0\end{array}\right],\left[\begin{array}{ll}0 & 0 \\ 1 & 0 \\ 0 & 0 \\ 0 & 0 \\ 0 & 0 \\ 0 & 0 \\ 0 & 0 \\ 0 & 0 \\ 0 & 0 \\ 0 & 1 \\ 0 & 0 \\ 0 & 0 \\ 1 & 0 \\ 0 & 0 \\ 0 & 0\end{array}\right]$

Obviously, the hypothesis in the above corollary holds when $m=1$, so that it includes the already known result in Proposition 4.4. Let us see that it includes also the other already known case, when the given system is controllable.

Proposition 7.3 Let $(J, B)$ be a controllable pair. Then the hypothesis in the above corollary holds, so that, the conclusions (1) and (2) are valid.

More explicitly:

(i) The (non-zero) Hermite indices $h$ are just the Segre characteristic p, up to permutations. In particular, the number of non-redundant controls are just $n$, so that one can assume $m=n$ and $h_{j}=p_{\sigma(j)}, \quad 1 \leq j \leq m=n$, where $\sigma$ is a permutation of $\{1, \ldots, n\}$.

In addition, $p_{1}=q_{1}=h_{1}$, so that $\sigma(1)=1$.

(ii) For each $1 \leq j \leq m=n$, there is only one block index $i_{j}(1)=\sigma(j)$ and one diagonal index $k_{j}(1)=h_{j}$.

In particular, $i_{1}(1)=1$ and $k_{1}(1)=p_{1}$.

(iii) The matrix $U$ has one non-zero block in each block column and block row, and they are identity blocks:

$$
U_{\sigma(j), j}=\left(I^{p_{\sigma(j)}} \mid 0\right) \in M_{p_{\sigma(j)} \times q_{j}}(\mathbb{C}) \quad \text { for } \quad 1 \leq j \leq m=n
$$

In particular, $\quad U_{11}=I^{p_{1}}$ 
Proof. If $(J, B)$ is controllable, the matrix in Lemma 5.5 is just $J$, up to permutations of the Jordan blocks, and $(i)$ follows.

Therefore $U_{i_{j}(1), j}$ is an identity block, so that $(i i)$ and (iii) follow from $\left(2^{\prime}\right)$ in Proposition 2.13.

Example 7.4 If $(J, B)$ is controllable with $p=(6,3,2), q=(6,5,4), h=(6,2,3)$, then:
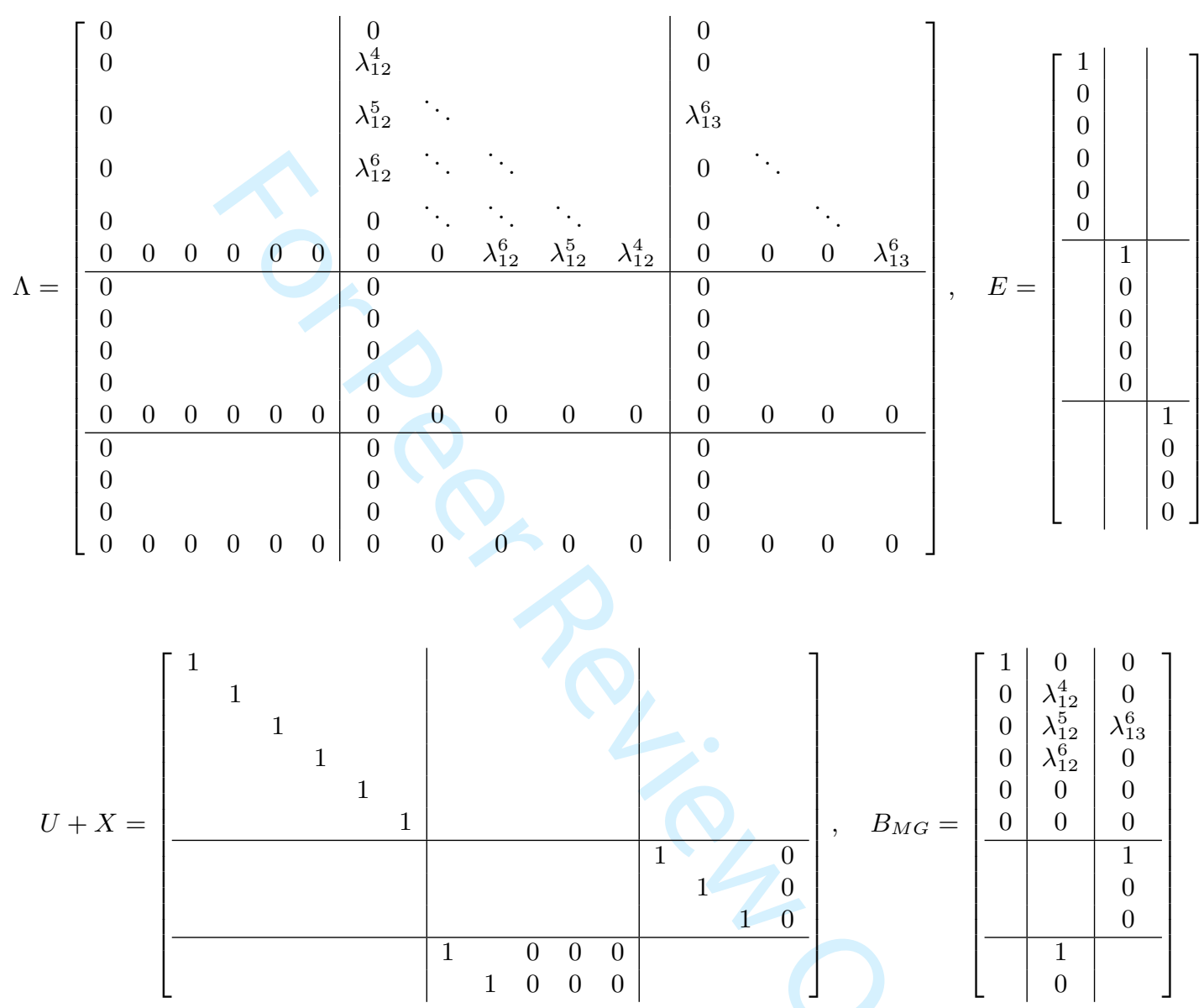

\section{The case $X \neq 0$}

When $X \neq 0$ further row BLD-ETs can be considered in order to simplify $X$, preserving $U$. In some particular cases, this simplification is not possible, so that $(U+X)(I-\Lambda)^{-1} E$ is indeed a canonical form. For example in the following obvious case, when $X$ has only a non-zero block in each block column and it is a diagonal one.

Proposition 8.1 In the conditions of Corollary 7.1, let us assume $X \neq 0$ but it has only a non-zero block in each column block, which is a diagonal one. That is to say, for each block column $X_{* j}, 2 \leq j \leq m$, there is a unique row block index $i[j]$ (among those having a non-zero block of $U$ in some left block column)

$$
i[j] \in \cup_{1 \leq u<j}\left\{i_{u}(1), \ldots, i_{u}\left(r_{u}\right)\right\} \quad \text { and } \quad l_{j} \leq p_{i[j]}, q_{j}
$$


such that $X_{i[j], j}$ is the only non-zero block in $X_{* j}$ and it is a diagonal one:

$$
X_{i[j], j}=x_{i[j], j}^{l_{j}} I_{l_{j}} ; \quad X_{i, j}=0 \quad \text { if } \quad i \neq i[j]
$$

Then, the p-equivalence class of $B$ is characterized by:

(i) the heights $q_{1} \geq \cdots \geq q_{m}$,

(ii) the matrix $\Lambda$ of Hermite coefficients,

(iii) the m-monogenic indices $i_{j}(1), \ldots, i_{j}\left(r_{j}\right), \quad k_{j}(1), \ldots, k_{j}\left(r_{j}\right), \quad 1 \leq j \leq m$

(iv) the row indices $i[j]$, the heights $l_{j}$, and the coefficients $x_{i[j], j}^{l_{j}}$ for $1 \leq j \leq m$

and a p-canonical form of $B$ is:

$$
B_{M G}=(U+X)(I-\Lambda)^{-1} E
$$

Example 8.2 Let us consider $p=(8,4,3)$ as in Example 7.2, but $B$ as below. Then we obtain:

$$
B=\left[\begin{array}{c|c}
0 & 0 \\
1 & 0 \\
2 & 0 \\
-1 & -3 \\
0 & -4 \\
5 & 5 \\
1 & -1 \\
-1 & -14 \\
\hline 0 & 0 \\
3 & 0 \\
0 & 1 \\
-1 & -7 \\
\hline 4 & -4 \\
5 & -3 \\
-1 & -10
\end{array}\right], \quad K=\left[\begin{array}{ccccccc|ccccc}
0 & 0 & 0 & 0 & 0 & 0 & 0 & 0 & 0 & 0 & 0 & 0 \\
1 & 0 & 0 & 0 & 0 & 0 & 0 & 0 & 0 & 0 & 0 & 0 \\
2 & 1 & 0 & 0 & 0 & 0 & 0 & 0 & 0 & 0 & 0 & 0 \\
-1 & 2 & 1 & 0 & 0 & 0 & 0 & -3 & 0 & 0 & 0 & 0 \\
0 & -1 & 2 & 1 & 0 & 0 & 0 & -4 & -3 & 0 & 0 & 0 \\
5 & 0 & -1 & 2 & 1 & 0 & 0 & 5 & -4 & -3 & 0 & 0 \\
1 & 5 & 0 & -1 & 2 & 1 & 0 & -1 & 5 & -4 & -3 & 0 \\
-1 & 1 & 5 & 0 & -1 & 2 & 1 & -14 & -1 & 5 & -4 & -3 \\
\hline 0 & 0 & 0 & 0 & 0 & 0 & 0 & 0 & 0 & 0 & 0 & 0 \\
3 & 0 & 0 & 0 & 0 & 0 & 0 & 0 & 0 & 0 & 0 & 0 \\
0 & 3 & 0 & 0 & 0 & 0 & 0 & 1 & 0 & 0 & 0 & 0 \\
-1 & 0 & 3 & 0 & 0 & 0 & 0 & -7 & 1 & 0 & 0 & 0 \\
\hline 4 & 0 & 0 & 0 & 0 & 0 & 0 & -4 & 0 & 0 & 0 & 0 \\
5 & 4 & 0 & 0 & 0 & 0 & 0 & -3 & -4 & 0 & 0 & 0 \\
-1 & 5 & 4 & 0 & 0 & 0 & 0 & -10 & -3 & -4 & 0 & 0
\end{array}\right]
$$

$$
\Lambda=\left[\begin{array}{ccccccc|ccccc}
0 & 0 & 0 & 0 & 0 & 0 & 0 & 0 & 0 & 0 & 0 & 0 \\
0 & 0 & 0 & 0 & 0 & 0 & 0 & 0 & 0 & 0 & 0 & 0 \\
0 & 0 & 0 & 0 & 0 & 0 & 0 & -3 & 0 & 0 & 0 & 0 \\
0 & 0 & 0 & 0 & 0 & 0 & 0 & 2 & -3 & 0 & 0 & 0 \\
0 & 0 & 0 & 0 & 0 & 0 & 0 & 0 & 2 & -3 & 0 & 0 \\
0 & 0 & 0 & 0 & 0 & 0 & 0 & 0 & 0 & 2 & -3 & 0 \\
0 & 0 & 0 & 0 & 0 & 0 & 0 & 0 & 0 & 0 & 2 & -3 \\
\hline 0 & 0 & 0 & 0 & 0 & 0 & 0 & 0 & 0 & 0 & 0 & 0 \\
0 & 0 & 0 & 0 & 0 & 0 & 0 & 0 & 0 & 0 & 0 & 0 \\
0 & 0 & 0 & 0 & 0 & 0 & 0 & 0 & 0 & 0 & 0 & 0 \\
0 & 0 & 0 & 0 & 0 & 0 & 0 & 0 & 0 & 0 & 0 & 0 \\
0 & 0 & 0 & 0 & 0 & 0 & 0 & 0 & 0 & 0 & 0 & 0
\end{array}\right], K_{H}^{\prime}=\left[\begin{array}{cccccccc|ccccc}
0 & 0 & 0 & 0 & 0 & 0 & 0 & 0 & 0 & 0 & 0 & 0 \\
1 & 0 & 0 & 0 & 0 & 0 & 0 & 0 & 0 & 0 & 0 & 0 \\
2 & 1 & 0 & 0 & 0 & 0 & 0 & 0 & 0 & 0 & 0 & 0 \\
-1 & 2 & 1 & 0 & 0 & 0 & 0 & 0 & 0 & 0 & 0 & 0 \\
0 & -1 & 2 & 1 & 0 & 0 & 0 & 0 & 0 & 0 & 0 & 0 \\
5 & 0 & -1 & 2 & 1 & 0 & 0 & -2 & 0 & 0 & 0 & 0 \\
1 & 5 & 0 & -1 & 2 & 1 & 0 & 1 & -2 & 0 & 0 & 0 \\
-1 & 1 & 5 & 0 & -1 & 2 & 1 & 1 & 1 & -2 & 0 & 0 \\
\hline 0 & 0 & 0 & 0 & 0 & 0 & 0 & 0 & 0 & 0 & 0 & 0 \\
3 & 0 & 0 & 0 & 0 & 0 & 0 & 0 & 0 & 0 & 0 & 0 \\
0 & 3 & 0 & 0 & 0 & 0 & 0 & 1 & 0 & 0 & 0 & 0 \\
-1 & 0 & 3 & 0 & 0 & 0 & 0 & 2 & 1 & 0 & 0 & 0 \\
\hline 4 & 0 & 0 & 0 & 0 & 0 & 0 & -4 & 0 & 0 & 0 & 0 \\
5 & 4 & 0 & 0 & 0 & 0 & 0 & -3 & -4 & 0 & 0 & 0 \\
-1 & 5 & 4 & 0 & 0 & 0 & 0 & 2 & -3 & -4 & 0 & 0
\end{array}\right]
$$




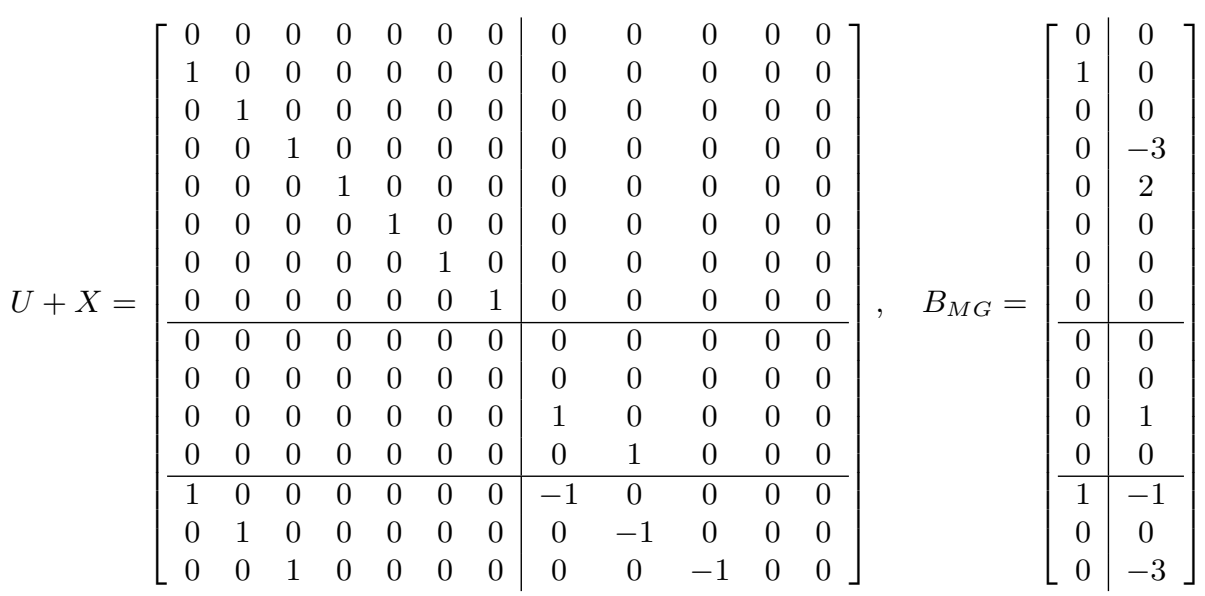

For more general $X \neq 0$, some (non trivial) reductions are possible but not always MG canonical forms are attempted. Let us consider $m=2$, in which case Theorem 6.1 says:

Corollary 8.3 Let $J \in M_{N}(\mathbb{C})$ be a nilpotent lower Jordan matrix with Segre characteristic $p=$ $\left(p_{1}, \ldots, p_{n}\right), B=\left(b_{1}, b_{2}\right) \in M_{N \times 2},\left(h_{1}, h_{2}\right)$ be its Hermite indices, and $B_{M G}=(U+X)(I-\Lambda)^{-1} E$ be its $M G$ reduced form (see Definition 6.4). More explicitly,

- $U+X$ is characterized as follows:

(1) For $j=1,2$ :

$$
\begin{gathered}
U_{i j}=0 \quad \text { if } \quad i \neq i_{j}(1), \ldots, i_{j}\left(r_{j}\right) \\
U_{i_{j}(s), j}=I_{i_{j}(s), j}^{k_{j}(s)}, \quad 1 \leq s \leq r_{j}
\end{gathered}
$$

where the finite sequences of block indices $i_{j}(1), \ldots, i_{j}\left(r_{j}\right)$ and diagonal indices $k_{j}(1), \ldots, k_{j}\left(r_{j}\right)$ satisfy:

$$
\begin{gathered}
\left\{i_{1}(1), \ldots, i_{1}\left(r_{1}\right)\right\} \cap\left\{i_{2}(1), \ldots, i_{2}\left(r_{2}\right)\right\}=\emptyset \\
1 \leq i_{j}(1)<\ldots<i_{j}\left(r_{j}\right) \leq n \\
h_{j}=k_{j}(1)>\ldots>k_{j}\left(r_{j}\right) \geq 1 \\
p_{i_{j}(s)} \geq k_{j}(s), \quad 1 \leq s \leq r_{j} \\
p_{i_{j}(s+1)}-k_{j}(s+1)<p_{i_{j}(s)}-k_{j}(s), \quad \text { for } 1 \leq s<r_{j}
\end{gathered}
$$

(2) $X_{* 1}=0 ; \quad X_{i 2}=0$ if $i \neq i_{1}(s)$ for $1 \leq s \leq r_{1}$.

If $X_{i_{1}(s), 2}=\sum_{l=1}^{l_{i_{1}(s)}} x_{i_{1}(s)}^{l} I_{i_{1}(s), 2}^{l} \neq 0$, its height $l_{i_{1}(s)}$ satisfies

$$
p_{i_{1}(s)}-l_{i_{1}(s)}<p_{i_{2}(t)}-k_{2}(t) \quad \text { or } \quad l_{i_{1}(s)}>k_{2}(t) \quad \text { for } \quad \text { all } \quad 1 \leq t \leq r_{2}
$$

- $B_{M G}=(U+X)(I+\Lambda) H$ because $\Lambda^{2}=0$ and then $(I-\Lambda)^{-1}=I+\Lambda$.

Let us see when a non-zero block $X_{i(s), 2}$ can be cancelled by means of additional BLD-ETs preserving the unitary blocks. 
Lemma 8.4 Let $J \in M_{N}(\mathbb{C})$ be a nilpotent lower Jordan matrix, $B=\left(b_{1}, b_{2}\right) \in M_{N \times 2}$ and $B_{M G}=(U+X)(I+\Lambda) E$ be its $M G$ reduced form. Let $i=i_{1}(s), j=i_{1}(t), s \neq t$ for any $1 \leq s, t \leq r_{1}$.

Then, we can make $X_{j, 2}=0$ (without modifying the other blocks) by means of row BLD-ETs using $x_{i}^{l_{i}}$ if

$$
l_{j} \leq \min \left(l_{i}, l_{i}+p_{j}-p_{i}, l_{i}+k_{j}-k_{i}-1\right)
$$

and it is not possible if $l_{j}>l_{i}+k_{j}-k_{i}$.

Proof.

We can remove $x_{j}^{l_{j}}$ using $x_{i}^{l_{i}}$, provided that the heights and depths comply $l_{j} \leq l_{i}, \quad p_{j}-l_{j} \geq$ $p_{i}-l_{i}$ : by adding the first $p_{i}-l_{i}+l_{j}$ rows of $(U+X)_{i *}$ multiplied by $\alpha=-x_{j}^{l_{j}} / x_{i}^{l_{i}}$ to the last rows of $(U+X)_{j *}$. The obtained block $(U+X)_{j *}(1)$ will be:

- $U_{j, 1}(1)=U_{j, 1}+\alpha I_{j, 1}^{k_{i}-l_{i}+l_{j}} \quad$ or $\quad U_{j, 1}(1)=U_{j, 1} \quad$ if $\quad l_{j} \leq l_{i}-k_{i}$

- $x_{j}^{s}(1)=x_{j}^{s}+\alpha x_{i}^{s+l_{i}-l_{j}} \quad$ if $\quad s \leq p_{i}-l_{i}+l_{j}$

- $x_{j}^{s}(1)=x_{j}^{s} \quad$ otherwise

Notice that $x_{j}^{s}(1)=x_{j}^{s}$ if $s>l_{j}$ because $s+l_{i}-l_{j}>l_{i}$. Then, the height of $X_{j, 2}(1)$ is less than $l_{j}$.

If $l_{j} \leq l_{i}-k_{i}$ we have that $U_{j, 1}(1)=U_{j, 1}$, the height of $X_{j, 2}(1)$ is less than $l_{j}$ and the other blocks do not change.

If $l_{i}-k_{i}<l_{j}<k_{j}+l_{i}-k_{i}$, in order to remove $\alpha$ in $U_{j, 1}(1)$, we add the first $p_{j}-k_{j}+k_{i}-l_{i}+l_{j}$ rows of $(U+X)_{j *}(1)$ multiplied by $-\alpha$ to the last rows of itself. The obtained block $(U+X)_{j *}(2)$ will be:

- $U_{j, 1}(2)=U_{j, 1}-\alpha^{2} I_{j, 1}^{2\left(k_{i}-l_{i}+l_{j}\right)-k_{j}} \quad$ or $\quad U_{j, 1}(2)=U_{j, 1} \quad$ if $\quad 2\left(k_{i}-l_{i}+l_{j}\right) \leq k_{j}$

- $x_{j}^{s}(2)=x_{j}^{s}(1)-\alpha x_{j}^{s+k_{j}-k_{i}+l_{i}-l_{j}}(1) \quad$ if $\quad s \leq p_{j}-k_{j}+k_{i}-l_{i}+l_{j}$

- $x_{j}^{s}(2)=x_{j}^{s}(1) \quad$ otherwise.

Notice that $s+k_{j}-k_{i}+l_{i}-l_{j}>l_{j}$ if $s \geq l_{j}$, so that $x_{j}^{s}(2)=x_{j}^{s}(1)=0$. Then, this second reduction does not increase the height of $X_{j, 2}(1)$ and the height of $-\alpha^{2}$ in $U_{j, 1}(2)$ is less than the height of $\alpha$ in $U_{j, 1}(1)$.

By recurrence, after a finite number of steps, the non-zero entry $(-1)^{k+1} \alpha^{k}$ of the unit block will disappear.

Also, by recurrence, in a finite number of steps $X_{j, 2}$ becomes 0 .

Finally, if $l_{j}>k_{j}+l_{i}-k_{i}$, the height of the entry $\alpha$ in the block $U_{j, 1}(1)$ obtained in the first step will be greater than $k_{j}$ and it can only be eliminated by returning to the initial situation. 
By using the above lemma recurrently as in the MG-algorithm, we reduce the number of non-zero blocks of $X$ preserving the other ones and we obtain a matrix $X^{\prime}$.

Proposition 8.5 Let $J \in M_{N}(\mathbb{C})$ be a nilpotent lower Jordan matrix, $B=\left(b_{1}, b_{2}\right) \in M_{N \times 2}$ and let $B_{M G}=(U+X)(I+\Lambda) E$ be its $M G$ reduced form.

Let $X_{i_{1}(s), 2}, 1 \leq s \leq r_{1}$, be the possible non-zero blocks of $X$. Then, by means of row BLD-ETs preserving $U$, we can reduce the non-zero blocs of $X$ to the ones which correspond to indices $1 \leq s_{1}<\cdots<s_{r_{1}^{\prime}}$ characterized by:

(1) $i_{1}(1) \leq i_{1}\left(s_{1}\right)<\ldots<i_{1}\left(s_{r_{1}^{\prime}}\right) \leq i_{1}\left(r_{1}\right)$

(2) $l_{i_{1}\left(s_{1}\right)}>\ldots>l_{i_{1}\left(s_{r_{1}^{\prime}}\right)} \geq 1$

(3) $p_{i_{1}\left(s_{j+1}\right)}-l_{i_{1}\left(s_{j+1}\right)}<p_{i_{1}\left(s_{j}\right)}-l_{i_{1}\left(s_{j}\right)}, \quad 1 \leq j<r_{1}^{\prime}$

We recall that $\quad p_{i_{1}\left(s_{j}\right)}-l_{i_{1}\left(s_{j}\right)}<p_{i_{2}(t)}-k_{2}(t) \quad$ or $\quad l_{i_{1}\left(s_{j}\right)}>k_{2}(t) \quad$ for $\quad$ all $\quad 1 \leq t \leq r_{2}$.

The block indices $i_{1}\left(s_{j}\right), 1 \leq j \leq r_{1}^{\prime}$, as well as the heights $l_{i_{1}\left(s_{j}\right)}$ and the highest coefficients $x_{i_{1}\left(s_{j}\right)}^{l_{i_{1}}\left(s_{j}\right)}$, are invariant with regard to row BLD-ETs preserving $U$.

\section{Proof.}

Let $L_{1}=\max \left\{l_{i_{1}(s)}: 1 \leq s \leq r_{1}\right\}$ and $s_{1}=\max \left\{s: l_{i_{1}(s)}=L_{1}, 1 \leq s \leq r_{1}\right\}$.

If $s<s_{1}, l_{i_{1}(s)} \leq \min \left(l_{i_{1}\left(s_{1}\right)}, l_{i_{1}\left(s_{1}\right)}+p_{i_{1}(s)}-p_{i_{1}\left(s_{1}\right)}, l_{i_{1}\left(s_{1}\right)}+k_{1}(s)-k_{1}\left(s_{1}\right)-1\right)$ and from Lemma 8.4, by using $x_{i_{1}\left(s_{1}\right)}, X_{i(s)}$ becomes 0 .

If $s>s_{1}, l_{i_{1}(s)}<l_{i_{1}\left(s_{1}\right)}$ and, $p_{i_{1}(s)}-l_{i_{1}(s)} \geq p_{i_{1}\left(s_{1}\right)}-l_{i_{1}\left(s_{1}\right)}$ together with $p_{i_{1}\left(s_{1}\right)}-k_{1}\left(s_{1}\right)>$ $p_{i_{1}(s)}-k_{1}(s)$ implies that $k_{1}\left(s_{1}\right)-l_{i_{1}\left(s_{1}\right)}<k_{1}(s)-l_{i_{1}(s)}$. Then, the condition of Lemma 8.4 in order to do that $X_{i(s)}$ becomes 0 using $x_{i_{1}\left(s_{1}\right)}$ is given except for the case $p_{i_{1}(s)}-l_{i_{1}(s)}<p_{i_{1}\left(s_{1}\right)}-l_{i_{1}\left(s_{1}\right)}$.

By recurrence, we obtain the announced finite sequence.

Finally, let us eliminate some entries in the matrix $X^{\prime}$ obtained above:

Lemma 8.6 Let $J \in M_{N}(\mathbb{C})$ be a nilpotent lower Jordan matrix, $B=\left(b_{1}, b_{2}\right) \in M_{N \times 2}, B_{M G}=$ $(U+X)(I+\Lambda) E$ be its $M G$ reduced form, and $X^{\prime}$ be as in Proposition 8.5.

Let us consider $i_{1}\left(s_{j}\right), 1 \leq s_{j} \leq r_{1}^{\prime}$, and $l<l_{i_{1}\left(s_{j}\right)}$.

(1) Let us assume that there is $s_{i} \in\left\{s_{1}, \ldots, s_{r_{1}^{\prime}}\right\}$ such that some of the following conditions is satisfied

(i) $l \leq \min \left(l_{i_{1}\left(s_{i}\right)}-k_{1}\left(s_{i}\right), l_{i_{1}\left(s_{i}\right)}+p_{i_{1}\left(s_{j}\right)}-p_{i_{1}\left(s_{i}\right)}\right)$

(ii) $l_{i_{1}\left(s_{i}\right)}-k_{1}\left(s_{i}\right)<l \leq \min \left(l_{i_{1}\left(s_{i}\right)}, l_{i_{1}\left(s_{i}\right)}+p_{i_{1}\left(s_{j}\right)}-p_{i_{1}\left(s_{i}\right)}, l_{i_{1}\left(s_{i}\right)}+k_{1}\left(s_{j}\right)-k_{1}\left(s_{i}\right)-1\right)$ if $l_{i_{1}\left(s_{j}\right)}-k_{1}\left(s_{j}\right)<l_{i_{1}\left(s_{i}\right)}-k_{1}\left(s_{i}\right) \leq p_{i_{1}\left(s_{j}\right)}-k_{1}\left(s_{j}\right)$

(iii) $p_{i_{1}\left(s_{j}\right)}-k_{1}\left(s_{j}\right)<l_{i_{1}\left(s_{i}\right)}-k_{1}\left(s_{i}\right)<l \leq \min \left(l_{i_{1}\left(s_{i}\right)}, l_{i_{1}\left(s_{i}\right)}+p_{i_{1}\left(s_{j}\right)}-p_{i_{1}\left(s_{i}\right)}, l_{i_{1}\left(s_{i}\right)}+k_{1}\left(s_{j}\right)-\right.$ $\left.k_{1}\left(s_{i}\right)-1\right)$. 
Then, we can make $x_{i_{1}\left(s_{j}\right), 2}^{l}=0$, preserving $U$ and the blocks in $X$ other than $X_{i_{1}\left(s_{j}\right), 2}$, by means row BLD-ETs.

(2) Let us assume that there is $t \in\left\{1, \ldots, r_{2}\right\}$ such that

$$
l \leq \min \left(k_{2}(t), k_{2}(t)+p_{i_{1}\left(s_{j}\right)}-p_{i_{2}(t)}\right) .
$$

Then, we can make $x_{i_{1}\left(s_{j}\right), 2}^{l}=0$ by means of $\left(i_{2}(t), 2\right)$-row BLD-ETs preserving $U$ and the blocks in $X$ other than $X_{i_{1}\left(s_{j}\right), 2}$.

Proof.

This proof is very similar to the proof of Lemma 8.4.

For simplification, we write $j \equiv i_{1}\left(s_{j}\right), i \equiv i_{1}\left(s_{i}\right), k_{j} \equiv k_{1}\left(s_{j}\right), k_{i} \equiv k_{1}\left(s_{i}\right)$.

(1) We can remove $x_{j}^{l}$ using $x_{i}^{l_{i}}$, provided that the heights and depths comply $l \leq l_{i}, \quad p_{j}-l \geq$ $p_{i}-l_{i}$ : by adding the first $p_{i}-l_{i}+l$ rows of $(U+X)_{i *}$ multiplied by $\alpha=-x_{j}^{l} / x_{i}^{l_{i}}$ to the last rows of $(U+X)_{j *}$. The obtained block $(U+X)_{j *}(1)$ will be:

- $U_{j, 1}(1)=U_{j, 1}+\alpha I_{j, 1}^{k_{i}-l_{i}+l} \quad$ or $\quad U_{j, 1}(1)=U_{j, 1} \quad$ if $\quad l \leq l_{i}-k_{i}$

- $x_{j}^{s}(1)=x_{j}^{s}+\alpha x_{i}^{s+l_{i}-l}$ if $s \leq p_{i}-l_{i}+l$

- $x_{j}^{s}(1)=x_{j}^{s} \quad$ otherwise

Notice that $x_{j}^{s}(1)=x_{j}^{s}$ if $s>l$ because $s+l_{i}-l>l_{i}$.

If $l \leq l_{i}-k_{i}$ then, $x_{j}^{s}(1)=x_{j}^{s}$ if $s>l, x_{j}^{l}(1)=0$ and $U_{j, 1}(1)=U_{j, 1}$.

If $l_{i}-k_{i}<l<k_{j}+l_{i}-k_{i}$, in order to remove $\alpha$ in $U_{j, 1}(1)$, we will add the first $p_{j}-k_{j}+k_{i}-l_{i}+l$ rows of $(U+X)_{j *}(1)$ multiplied by $-\alpha$ to the last rows of itself. The obtained block $(U+X)_{j *}(2)$ will be:

- $U_{j, 1}(2)=U_{j, 1}-\alpha^{2} I_{j, 1}^{2\left(k_{i}-l_{i}+l\right)-k_{j}} \quad$ or $\quad U_{j, 1}(2)=U_{j, 1} \quad$ if $\quad 2\left(k_{i}-l_{i}+l\right) \leq k_{j}$

- $x_{j}^{s}(2)=x_{j}^{s}(1)-\alpha x_{j}^{s+k_{j}-k_{i}+l_{i}-l}(1) \quad$ if $\quad s \leq p_{j}-k_{j}+k_{i}-l_{i}+l$

- $x_{j}^{s}(2)=x_{j}^{s}(1) \quad$ otherwise.

Then, to guarantee that $x_{j}^{s}(2)=x_{j}^{s}(1)=0$ if $s \geq l$ it is necessary that $l_{j}-k_{j}<l_{i}-k_{i}$ if $l_{i}-k_{i} \leq p_{j}-k_{j}$. Then, this second reduction does not increase the height of $X_{j, 2}(1)$ and the height of $-\alpha^{2}$ in $U_{j, 1}(2)$ is less than the height of $\alpha$ in $U_{j, 1}(1)$.

By recurrence, after a finite number of steps, the non-zero entry $(-1)^{k+1} \alpha^{k}$ of the unit block will disappear.

Also, by recurrence, in a finite number of steps $X_{j, 2}$ becomes 0 .

Finally, if $l \geq k_{j}+l_{i}-k_{i}$, the height of the entry $\alpha$ in the block $U_{j, 1}(1)$ obtained in the first step will be greater than or equal to $k_{j}$ and it can only be eliminated by returning to the initial situation.

(2) It is obvious because the $\left(i_{2}(t), 2\right)$-row BLD-ETs affect only the block $X_{i_{1}\left(s_{j}\right), 2}$. 
Summarizing the above results, one obtains a more reduced form, but not always it is a canonical form (see Remark 8.8 and Example 8.9):

Theorem 8.7 Let $J \in M_{N}(\mathbb{C})$ be a nilpotent lower Jordan matrix with Segre characteristic $p=\left(p_{1}, \ldots, p_{n}\right), B=\left(b_{1}, b_{2}\right) \in M_{N \times 2}$ and $B_{M G}=(U+X)(I+\Lambda) E$ be its $M G$ reduced form. Then, $B$ is p-equivalent to the simplified matrix

$$
(U+\bar{X})(I+\Lambda) E
$$

where:

(1) There only non-zero blocks of $\bar{X}$ are $\bar{X}_{i_{1}\left(s_{j}\right)}, 1 \leq j \leq r_{1}^{\prime}$, corresponding to block indices $i_{1}\left(s_{j}\right)$ characterized by:

(i) $i_{1}(1) \leq i_{1}\left(s_{1}\right)<\ldots<i_{1}\left(s_{r_{1}^{\prime}}\right) \leq i_{1}\left(r_{1}\right)$

(ii) $l_{i_{1}\left(s_{1}\right)}>\ldots>l_{i_{1}\left(s_{r_{1}^{\prime}}\right)} \geq 1$

(iii) $p_{i_{1}\left(s_{j+1}\right)}-l_{i_{1}\left(s_{j+1}\right)}<p_{i_{1}\left(s_{j}\right)}-l_{i_{1}\left(s_{j}\right)}$, for $1 \leq j<r_{1}^{\prime}$

where $l_{i_{1}\left(s_{j}\right)}$ is the height of the corresponding block.

Both $i_{1}\left(s_{j}\right)$ and $l_{i_{1}\left(s_{j}\right)}, 1 \leq j \leq r_{1}^{\prime}$, are invariant with regard to row BLD-ETs preserving $U$.

(2) Their highest coefficients $x_{i_{1}\left(s_{j}\right)}^{l_{i_{1}}\left(s_{j}\right)}, 1 \leq j \leq r_{1}^{\prime}$, are also invariant with regard to row BLD-ETs preserving $U$.

(3) For $l<l_{i_{1}\left(s_{j}\right)}, 1 \leq j \leq r_{1}^{\prime}$ one has $x_{i_{1}\left(s_{j}\right)}^{l}=0$ if some of the following conditions is satisfied:

(i) $l \leq \min \left(l_{i_{1}\left(s_{i}\right)}-k_{1}\left(s_{i}\right), l_{i_{1}\left(s_{i}\right)}+p_{i_{1}\left(s_{j}\right)}-p_{i_{1}\left(s_{i}\right)}\right)$ for some $i \in \underline{r}_{1}^{\prime}$

(ii) $l_{i_{1}\left(s_{i}\right)}-k_{1}\left(s_{i}\right)<l \leq \min \left(l_{i_{1}\left(s_{i}\right)}, l_{i_{1}\left(s_{i}\right)}+p_{i_{1}\left(s_{j}\right)}-p_{i_{1}\left(s_{i}\right)}, l_{i_{1}\left(s_{i}\right)}+k_{1}\left(s_{j}\right)-k_{1}\left(s_{i}\right)-1\right)$ if $l_{i_{1}\left(s_{j}\right)}-k_{1}\left(s_{j}\right)<l_{i_{1}\left(s_{i}\right)}-k_{1}\left(s_{i}\right) \leq p_{i_{1}\left(s_{j}\right)}-k_{1}\left(s_{j}\right)$ for some $i \in \underline{r}_{1}^{\prime}$

(iii) $p_{i_{1}\left(s_{j}\right)}-k_{1}\left(s_{j}\right)<l_{i_{1}\left(s_{i}\right)}-k_{1}\left(s_{i}\right)<l \leq \min \left(l_{i_{1}\left(s_{i}\right)}, l_{i_{1}\left(s_{i}\right)}+p_{i_{1}\left(s_{j}\right)}-p_{i_{1}\left(s_{i}\right)}, l_{i_{1}\left(s_{i}\right)}+k_{1}\left(s_{j}\right)-\right.$ $\left.k_{1}\left(s_{i}\right)-1\right)$ for some $i \in \underline{r}_{1}^{\prime}$

(iv) $l \leq \min \left(k_{2}(t), k_{2}(t)+p_{i_{1}\left(s_{j}\right)}-p_{i_{2}(t)}\right)$ for some $t \in \underline{r}_{2}$

Remark 8.8 The coefficients $x_{i_{1}\left(s_{j}\right)}^{l}, l<l_{i_{1}\left(s_{j}\right)}, 1 \leq j \leq r_{1}^{\prime}$, which do not satisfy any of the conditions in (3) above, can not be invariant with regard to row BLD-ETs preserving $U$. So, the reduced form $(U+\bar{X})(I+\Lambda) E$ in Theorem 8.7 is not canonical. See the following example:

Example 8.9 Let $J \in M_{N}(\mathbb{C})$ be a nilpotent lower Jordan matrix with Segre characteristic $\left(p_{1}, p_{2}\right)=(5,3), B=\left(b_{1}, b_{2}\right) \in M_{N \times 2}$ and reduced form $(U+\bar{X})(I+\Lambda) E$. Let us consider some blocks of $U$ and $X: U_{11}, U_{21}, X_{12}, X_{22}$ with the indices $\left(k_{1}, k_{2}\right)=(3,2), \quad\left(l_{1}, l_{2}\right)=(4,3)$. This is to say that the first matrix below (writing only the first columns of each block) is a submatrix of $U+X$.

From Theorem 8.7, using $x_{2}^{3} \neq 0$ and $x_{1}^{4} \neq 0$, only $x_{1}^{1}$ and $x_{2}^{1}$ can become 0 . We can assume that they are always 0 . 
We will try that $x_{1}^{3}$ become 0 using $x_{2}^{3}$ because they meet the conditions of heights and depths:

$$
\left[\begin{array}{c|c}
0 & 0 \\
0 & x_{1}^{4} \\
1 & x_{1}^{3} \\
0 & x_{1}^{2} \\
0 & 0 \\
\hline 0 & x_{2}^{3} \\
1 & x_{2}^{2} \\
0 & 0
\end{array}\right] \longrightarrow\left[\begin{array}{c|c}
0 & 0 \\
0 & x_{1}^{4} \\
1 & 0 \\
\alpha & x_{1}^{2}(1) \\
0 & 0 \\
\hline 0 & x_{2}^{3} \\
1 & x_{2}^{2} \\
0 & 0
\end{array}\right] \longrightarrow\left[\begin{array}{c|c}
0 & 0 \\
0 & x_{1}^{4} \\
1 & -\alpha x_{1}^{4} \\
0 & x_{1}^{2}(1) \\
-\alpha^{2} & 0 \\
\hline 0 & x_{2}^{3} \\
1 & x_{2}^{2} \\
0 & 0
\end{array}\right] \longrightarrow\left[\begin{array}{c|c}
0 & 0 \\
0 & x_{1}^{4} \\
1 & -\alpha x_{1}^{4} \\
0 & x_{1}^{2}(2) \\
0 & 0 \\
\hline 0 & x_{2}^{3} \\
1 & x_{2}^{2} \\
0 & 0
\end{array}\right]
$$

where $\alpha=-x_{1}^{3} / x_{2}^{3}, x_{1}^{2}(1)=x_{1}^{2}+\alpha x_{2}^{2}, x_{1}^{2}(2)=x_{1}^{2}(1)+\alpha^{2} x_{1}^{4}=x_{1}^{2}+\alpha x_{2}^{2}+\alpha^{2} x_{1}^{4}$.

We see that we have not succeeded and, on the other hand, we have obtained a different reduced form with the same number of parameters.

\section{References}

[1] A.Compta; J. Ferrer, Geometric classification of monogenic subspaces and uniparametric linear control systems, Linear and multilinear algebra, 63 - 9 (2015), p. 1768-1785.

[2] A. Compta; J. Ferrer; M. Peña, Perturbed marked reduced forms of invariant subspaces, Linear Algebra Appl., 559 (2018), p. 194-226.

[3] D. Hinrichsen; D. Prätzel-Wolters, A Jordan canonical form for reachable linear systems, Linear Algebra Appl., 122-124 (1989), p. 489-524.

[4] I. Kaplansky, Infinite Abelian Groups. Ann Arbor (MI): University of Micihigan Pres; 1954.

[5] C.M. Ringel; M. Schmidmeier, Invariant subspaces of nilpotent operators. I., Journal Reine Angew. Math. Band, 614 (2008), p. 1-52.

[6] M.A. Shayman, On the variety of invariant subspaces of a finite-dimensional linear operator, Trans. Amer. Math. Soc., vol. 274 (2) (1982), p. 721-747. 